\title{
Homogenization of plasticity equations with two-scale convergence methods
}

\author{
B. Schweizer and M. Veneroni \\ Preprint 2013-12 \\ Oktober 2013
}

Fakultät für Mathematik

Technische Universität Dortmund

Vogelpothsweg 87

44227 Dortmund 



\title{
Homogenization of plasticity equations with two-scale convergence methods
}

\author{
B. Schweizer* and M. Veneroni ${ }^{\dagger}$
}

October 9, 2013

\begin{abstract}
We investigate the deformation of heterogeneous plastic materials. The model uses internal variables and kinematic hardening, elastic and plastic strain are used in an infinitesimal strain theory. For periodic material properties with periodicity length scale $\eta>0$, we obtain the limiting system as $\eta \rightarrow 0$. The limiting two-scale plasticity model coincides with well-known effective models. Our direct approach relies on abstract tools from two-scale convergence (regarding convex functionals and monotone operators) and on higher order estimates for solution sequences.
\end{abstract}

Keywords: plasticity, homogenization, two-scale convergence, convex analysis

MSC: 74C05, 74Q10, 35B27

\section{Introduction}

The effective behavior of heterogeneous plastic materials is of great interest in the applied sciences. Materials with periodic coefficients have been studied extensively in the engineering literature, the first mathematical formulation of an effective (twoscale) limit system seems to appear in [2]. Analytically, the rigorous derivation of an effective model (even for periodic materials) is not an easy task, results are quite recent, starting with $[28,29,20]$. All these investigations (and the present contribution) rely on the presence of some hardening effect. Regarding models without hardening, we are only aware of [13].

The analytical difficulties regard the flow-rule, often written with the subdifferential $\partial \Psi$ of some convex function $\Psi$ as $\partial_{t} p \in \partial \Psi(\sigma)$ (in this exposition we neglect hardening and use a flow-rule with the plastic strain $p$ as the only inner variable). In rateindependent material laws, $\Psi$ vanishes on a set of admissible stresses $\sigma$ and takes the value $+\infty$ outside this set (the von-Mises and the Tresca model are of this form). Accordingly, the (monotone) subdifferential is multi-valued. This degeneracy in the

*Technische Universität Dortmund, Fakultät für Mathematik, Vogelpothsweg 87, D-44227 Dortmund, Germany.

†Dipartimento di Matematica "F. Casorati", Università di Pavia, Via Ferrata 1, 27100 Pavia, Italy. 
flow-rule leads, on the one hand, to difficulties in the derivation of regularity properties and, on the other hand, to difficulties in the interpretation of the flow-rule. It is standard to use weak solution concepts and convex analysis tools to encode the flowrule with an energy inequality, exploiting the principle of maximal dissipation (in its most consequent form, this leads to the definition of energetic solutions for rateindependent processes, see $[16,18])$. Nevertheless, it is not an easy task to combine these concepts with the method of two-scale convergence.

One approach (mainly pushed forward by Visintin) is to exploit the variational structure of the flow-rule and to obtain homogenization results with two-scale convergence methods. In this approach, the behavior of functionals $\Psi=\Psi(\cdot ; x, y)$ in the context of two-scale convergence must be investigated: What is the behavior of $\Psi\left(u^{\eta}(x) ; x, x / \eta\right)$ when $u^{\eta}$ is two-scale convergent for $\eta \rightarrow 0$ ? Our approach uses the corresponding abstract results.

In many applications it is desirable to generalize the flow-rule to a law $\partial_{t} p \in g(\sigma)$, where $g$ is a maximal monotone operator which is not necessarily a subdifferential (in the periodic homogenization problem, $g=g\left(\sigma^{\eta}(x) ; x, x / \eta\right)$ must be investigated). The results of $[3,20]$ are obtained in this setting of maximal monotone operators. In their analysis, the authors introduce the method of phase-shift convergence, which has similarities with the method of periodic unfolding (see [11]). The authors use the method of two-scale convergence and, additionally, an auxiliary problem in which phase-shifted coefficients are used.

We present here a derivation of the well-known two-scale effective system with a direct two-scale convergence method; the limit system is as in [2], but we include second order time derivatives of the deformation. The limit procedure becomes possible by combining two-scale convergence results for functionals with higher order estimates (solutions have additional regularity in time). We emphasize that the additional time regularity that we exploit in our method is not obtained from the second order time derivative; our method can also be used in the quasi-static case. We obtain the linear laws of the effective model by a direct two-scale convergence argument. The effective two-scale flow-rule is obtained from an energy inequality (maximal dissipation principle) which, in turn, is obtained with the help of the linear laws and a lowersemicontinuity result for two-scale convergence in functionals. The central difficulty regards the two-scale features of $g$, which we encode in property (A4).

\section{The plasticity model with kinematic hardening}

We now describe the plasticity model that is investigated in this contribution. We follow the standard theory of infinitesimal strain plasticity, see e.g. [1, 15].

Elastic materials are usually described with a deformation $u: \Omega_{T}=\Omega \times(0, T) \rightarrow$ $\mathbb{R}^{n}$ and a stress tensor $\sigma: \Omega_{T} \rightarrow \mathbb{R}_{s}^{n \times n}$; here $\Omega \subset \mathbb{R}^{n}$ is the spatial domain, $n \in$ $\mathbb{N}$ the dimension, $(0, T)$ a time interval and $\mathbb{R}_{s}^{n \times n}$ the space of symmetric matrices. With the linear elasticity tensor $D \in \mathcal{L}\left(\mathbb{R}_{s}^{n \times n}, \mathbb{R}_{s}^{n \times n}\right)$, the material is described in the framework of linearized elasticity by Hooke's law $\sigma=D \nabla^{s} u$ and the conservation of linear momentum $\varrho \partial_{t}^{2} u-\nabla \cdot \sigma=f$. In these relations, $\nabla^{s} u=\left(\nabla u+(\nabla u)^{T}\right) / 2$ denotes the symmetrized gradient, $\varrho$ the density and $f$ the external forces.

Plastic materials are characterized by the fact that the stress-strain relation is no longer linear. Instead, some part $p$ of the deformation $\nabla^{s} u$ is realized by a plastic deformation; in consequence, only the elastic deformation tensor $\nabla^{s} u-p$ contributes 
to the forces, $\sigma=D\left(\nabla^{s} u-p\right)$. We investigate here the Prandtl-Reuss model of plasticity with kinematic hardening, including general internal variables $\xi$. In this model, the plastic deformation tensor $p$ can be recovered from the (in general) larger set of internal variables as $p=B \xi$. In every point $(x, t) \in \Omega \times(0, T)$ of space-time, the internal variables are $\xi(x, t) \in \mathbb{R}^{N}$ for some $N \in \mathbb{N}$. Accordingly, in every point of space-time, we demand that $B$ is a linear map, $B \in \mathcal{L}\left(\mathbb{R}^{N}, \mathbb{R}_{s}^{n \times n}\right)$. In many models, $B$ is assumed to be a projection to the first components of the vector of internal variables.

The flow-rule of plasticity is given by a nonlinear monotone (and usually multivalued) map $g$. Of special interest are the functions $g$ that are given by the von-Mises or the Tresca model. Without hardening parameter, the local change of internal variables depends only on the local stress $\sigma$. Since we want to define $g$ as a multivalued map $\mathbb{R}^{N} \rightarrow \mathbb{R}^{N}$ (same space in image and pre-image), we write $\partial_{t} \xi \in g\left(B^{T} \sigma\right)$. The hardening effect can be modelled with a positive map $L \in \mathcal{L}\left(\mathbb{R}^{N}, \mathbb{R}^{N}\right)$, using the modified flow-rule $\partial_{t} \xi \in g\left(B^{T} \sigma-L \xi\right)$.

\section{Periodic homogenization of plasticity problems}

The three relations (conservation of linear momentum, Hooke's law, and flow-rule) of the plasticity model are described above. We are interested in the case that the plastic material is highly heterogeneous. Assuming periodicity of the medium, we denote the length scale by $\eta>0$ and consider coefficient functions that depend (in a periodic way) on the fast variable $x / \eta$,

$$
D_{\eta}(x)=D\left(x, \frac{x}{\eta}\right), \quad L_{\eta}(x)=L\left(x, \frac{x}{\eta}\right), \quad g_{\eta}(\cdot ; x)=g\left(\cdot ; x, \frac{x}{\eta}\right),
$$

and, in the same way, $B_{\eta}(x)=B(x, x / \eta)$ and $\varrho_{\eta}(x)=\varrho(x, x / \eta)$. We assume that the functions have the periodicity of the unit cube $Y=[0,1]^{n}$ in the variable $y=x / \eta$.

Plasticity problem in the heterogeneous medium. With the oscillatory coefficients, the original problem (or $\eta$-problem) is the following: We search for $u^{\eta}: \Omega_{T} \rightarrow$ $\mathbb{R}^{n}, \sigma^{\eta}: \Omega_{T} \rightarrow \mathbb{R}_{s}^{n \times n}$ and $\xi^{\eta}: \Omega_{T} \rightarrow \mathbb{R}^{N}$ solving

$$
\begin{aligned}
\varrho_{\eta} \partial_{t}^{2} u^{\eta} & =\nabla \cdot \sigma^{\eta}+f & & \text { in } \Omega_{T}, \\
\sigma^{\eta} & =D_{\eta}\left(\nabla^{s} u^{\eta}-B_{\eta} \xi^{\eta}\right) & & \text { in } \Omega_{T}, \\
\partial_{t} \xi^{\eta} & \in g_{\eta}\left(B_{\eta}^{T} \sigma^{\eta}-L_{\eta} \xi^{\eta}\right) & & \text { in } \Omega_{T} .
\end{aligned}
$$

We understand (1.2a) in the sense of distributions on $\Omega_{T}$ (since $\varrho$ does not depend on $t$, the first term is well-defined in the distributional sense), the other relations are imposed pointwise. In Section 2, we complete system (1.2), as well as the following homogenized system, with suitable initial data and boundary conditions.

The homogenized problem. The plasticity problem in the homogenized medium ( $\eta$-independent coefficients) is a two-scale problem: The set of independent unknowns is enlarged by $y \in Y$. The homogenized or effective model is the following: We search for $u: \Omega_{T} \rightarrow \mathbb{R}^{n}, v: \Omega_{T} \times Y \rightarrow \mathbb{R}^{n}, w: \Omega_{T} \times Y \rightarrow \mathbb{R}^{N}$ and $z: \Omega_{T} \times Y \rightarrow \mathbb{R}_{s}^{n \times n}$ solving 


$$
\begin{aligned}
\bar{\varrho} \partial_{t}^{2} u & =\nabla \cdot\left(f_{Y} z d y\right)+f & & \text { in } \Omega_{T}, \\
z & =D\left(\nabla_{x}^{s} u+\nabla_{y}^{s} v-B w\right) & & \text { in } \Omega_{T} \times Y, \\
\nabla_{y} \cdot z & =0 & & \text { in } \Omega_{T} \times Y, \\
\partial_{t} w & \in g\left(B^{T} z-L w\right) & & \text { in } \Omega_{T} \times Y .
\end{aligned}
$$

In these equations, the two-scale stress is denoted by $z$, the inner variables by $w$; the variable $\bar{\varrho}$ denotes the effective density and is given by the arithmetic average $\bar{\varrho}(x)=f_{Y} \varrho(x, y) d y$. The unknown $v$ and equation (1.3c) were not present in system (1.2); they are typical features of two-scale homogenized systems and carry information on the limiting behavior of the space derivatives of $u^{\eta}$ and of $\sigma^{\eta}$ (see Lemma 3.1 below). Equation (1.3a) must be satisfied in the sense of distributions on $\Omega_{T}$, equation (1.3c) in the sense of distributions on $\Omega_{T} \times Y$, the other two relations are imposed pointwise on $\Omega_{T} \times Y$.

\section{Further comparison with the literature}

In the homogenization results [29] and [32] of Visintin, the maximal monotone operator $g$ is a subdifferential and kinematic hardening is used. Formally, $g=g(\cdot ; x, y)=$ $\partial \Psi(\cdot ; x, y)$ is assumed to be independent of $x$, but a remark notes that an additional $x$-dependence can also be treated. The interest in these contributions is in viscoplasticity, hence the case that $g$ is the subdifferential of an indicatrix function is not treated. Instead, a discussion of the homogenization process in the case $g=\partial \varphi$, where $\varphi$ is the indicatrix function of a convex set $K(y)$, is contained in [28].

The latter situation, in which $g$ is the subdifferential of an indicatrix, is also the subject in the homogenization result of [25]. In that contribution, strong solutions are obtained with a Galerkin scheme. The homogenization proof, which is based on Tartar's energy method, uses appropriate oscillating test-functions provided by the Galerkin approximations.

In [20] and [3], the general maximal monotone multi-valued map $g$ does not depend on $x$. Since the approach uses the method of phase-shift convergence, it is not clear how to generalize the proof to $x$-dependent maps $g$.

Our result uses the property (A4) on $g$ to obtain the homogenization result (see Section 3.2). We observe in Lemma 3.3 that (A4) is satisfied when $g$ is a subdifferential (possibly, of an indicatrix function). In Lemma 3.4 we note that (A4) is satisfied if $g$ is maximal monotone and piecewise $x$-independent. We therefore obtain a result that is stronger than those mentioned above. Moreover, our assumption (A4) clarifies the problems with general $(x, y)$-dependent maximal monotone maps $g$ : utilizing maximal monotonicity in function spaces is limited by the fact that test-functions must be admissible in the sense of two-scale convergence. Indeed, the properties of maximal monotone operators in periodic homogenization problems require careful analysis, and we additionally refer to $[6,9,10,12,14]$.

The stochastic homogenization of the system was treated in [24] in one space dimension. In this stochastic setting, a problem appears that is closely related to the problems here: for $g=g(x, y)$, the weak convergence of a sequence of functions $g^{\eta}(x)=g\left(x, \chi^{\eta}(x)\right)$ must be analyzed. This has been possible in [24] with the notion 
of two-scale ergodicity. We emphasize that one space dimension permits control of the stress and is therefore very different from the higher dimensional case. Stochastic homogenization of subdifferential inclusions was analyzed in [26].

A related homogenization result is contained in [31], but it does not concern plasticity in our sense. The emphasis in [2] is on the interface conditions along (microscopic) interfaces. A gradient theory of plasticity was investigated in [21] using the framework of periodic unfolding. The homogenization for rate-independent systems has been developed in $[17,19]$, for a porous-media application see [23]. The homogenization of plasticity equations without hardening effect is treated in [13].

\section{Main results}

Our main result states that system (1.3) is the limit system for (1.2) in the sense of homogenization. In particular, for solutions of the two systems, there holds $u^{\eta} \rightarrow u$ in $L^{2}\left(\Omega_{T} ; \mathbb{R}^{n}\right)$ as $\eta \rightarrow 0$. The proof is based on two-scale convergence and monotonicity methods. Since solution concepts and function spaces must be clarified, we give a precise description of the results in this section.

\section{Existence result and estimates for plasticity equations}

Assumptions on the geometry, initial and boundary data. Let $\Omega \subset \mathbb{R}^{n}$ be a bounded Lipschitz domain, we restrict our considerations to domains with polygonal boundaries. Let $Y:=[0,1]^{n}$ be the unit cube, equipped with the topology of the $n$ dimensional torus. In particular, we can identify functions on $Y$ with their $Y$-periodic extensions; this identification is used in the definition of the space $H_{\sharp}^{1}(Y)$ of periodic functions and in the space of test functions $\mathcal{D}(\Omega \times Y)$.

We complement system (1.2) with the initial data and boundary conditions

$$
\begin{array}{rrl}
u^{\eta}(\cdot, 0)=u_{0}^{\eta}, & \partial_{t} u^{\eta}(\cdot, 0)=u_{1}^{\eta}, \quad \xi^{\eta}(\cdot, 0)=\xi_{0}^{\eta} & \text { on } \Omega, \\
u^{\eta}=U_{0}^{\eta} & \text { on } \partial \Omega \times(0, T) .
\end{array}
$$

Accordingly, we complement system (1.3) with the initial data and boundary conditions

$$
\begin{aligned}
u(\cdot, 0)=u_{0}, & \partial_{t} u(\cdot, 0)=u_{1} & & \text { on } \Omega, \\
w(\cdot, 0) & =w_{0} & & \text { on } \Omega \times Y, \\
u & =U_{0} & & \text { on } \partial \Omega \times(0, T) .
\end{aligned}
$$

Assumptions on coefficients. Let the coefficients satisfy, for bounds $\alpha, \beta>0$ :

(A1) The maps $D: \Omega \times Y \rightarrow \mathcal{L}\left(\mathbb{R}_{s}^{n \times n}, \mathbb{R}_{s}^{n \times n}\right), B: \Omega \times Y \rightarrow \mathcal{L}\left(\mathbb{R}^{N}, \mathbb{R}_{s}^{n \times n}\right), L: \Omega \times Y \rightarrow$ $\mathcal{L}\left(\mathbb{R}^{N}, \mathbb{R}^{N}\right)$, and $\varrho: \Omega \times Y \rightarrow[\alpha, \beta]$ are of Caratheodory type: continuous in $x$ for almost every $y \in Y$ and measurable in $y$ for every $x \in \Omega$. We assume the symmetries $D=D^{T}$ and $L=L^{T}$ (with respect to the scalar product of matrices), and the positivities

$$
\alpha\|\sigma\|^{2} \leq D^{-1}(x, y) \sigma: \sigma \leq \beta\|\sigma\|^{2}, \quad \alpha\|\xi\|^{2} \leq L(x, y) \xi: \xi \leq \beta\|\xi\|^{2}
$$

for a.e. $(x, y) \in \Omega \times Y$, every $\sigma \in \mathbb{R}_{s}^{n \times n}$ and every $\xi \in \mathbb{R}^{N}$. 
(A2) Let $g: \mathbb{R}^{N} \times \Omega \times Y \rightarrow \mathcal{P}\left(\mathbb{R}^{N}\right)$ be a map such that the multi-valued map $g(\cdot ; x, y)$ : $\mathbb{R}^{N} \rightarrow \mathbb{R}^{N}$ is maximal monotone with $0 \in g(0 ; x, y)$. We assume measurability of the resolvent mapping $(x, y) \mapsto(\lambda \mathrm{id}+g(\cdot ; x, y))^{-1}(z)$ for every $z \in \mathbb{R}^{N}$.

(A3) For the existence result of Theorem 2.1 we assume that $g$ can be regularized: We assume that there exists a sequence of functions $g_{\delta}$ with $\delta \rightarrow 0$ such that, for a closed convex set $\{0\} \in K_{0} \subset \mathbb{R}^{N}$ and a constant $C_{g}>0$ independent of $\delta>0$,

$$
\begin{array}{ll}
g_{\delta}: \mathbb{R}^{N} \times \Omega \times Y \rightarrow \mathbb{R}^{N} & \text { is locally Lipschitz and monotone in } \xi, \\
\left|g_{\delta}(\xi ; x, y)\right| \leq C_{g} & \forall \xi \in K_{0} .
\end{array}
$$

For every pair $f, h \in L^{2}(\Omega \times Y)$ with $h \in g(f)$, there exists a sequence $f_{\delta}$ such that, as $\delta \rightarrow 0$,

$$
f_{\delta} \rightarrow f, \quad g_{\delta}\left(f_{\delta}\right) \rightarrow h \quad \text { strongly in } L^{2}(\Omega \times Y) .
$$

We give some remarks on the above assumptions. In the Caratheodory assumption of (A1), the roles of $x$ and $y$ can also be exchanged. Furthermore, continuity can also be relaxed to a Borel-measurability assumption. This allows, in particular, piecewise continuous functions (relative to a finite number of Borel-measurable subdomains). All these functions are, in particular, admissible test-functions in the sense of two-scale convergence.

The monotonicity assumption of (A2) can be written as

$$
(\tilde{\zeta}-\zeta) \cdot(\tilde{\xi}-\xi) \geq 0 \quad \forall \xi, \tilde{\xi} \in \mathbb{R}^{N}, \zeta \in g(\xi ; x, y), \tilde{\zeta} \in g(\tilde{\xi} ; x, y)
$$

for arbitrary $(x, y) \in \Omega \times Y$. Maximality is meant with respect to graph inclusion; by a well known result (see [8]), maximality of $g$ is equivalent to the invertibility of $\lambda$ id $+g$ for some $\lambda>0$. Due to the normalization of $g$ there holds $\zeta \cdot \xi \geq 0$ for every $\zeta \in g(\xi ; x, y)$.

We note that the maximal monotonicity of $g(\cdot ; x, y): \mathbb{R}^{N \rightarrow} \mathbb{R}^{N}$ implies the maximal monotonicity of the induced map $g_{\Omega \times Y}: L^{2}(\Omega \times Y) \rightarrow L^{2}(\Omega \times Y)$. This can be seen as follows: For $\lambda>0$, the map $\lambda \mathrm{id}+g_{\Omega \times Y}$ is pointwise invertible by maximal monotonicity of the pointwise function. The pointwise defined inverse is a well-defined map $L^{2}(\Omega \times Y) \rightarrow L^{2}(\Omega \times Y)$ due to the measurability assumption and the fact that $j_{\lambda}$ is a contraction with $j_{\lambda}(0)=0$. We conclude from the characterization of maximal monotone operators that $g_{\Omega \times Y}: L^{2}(\Omega \times Y) \rightarrow L^{2}(\Omega \times Y)$ is maximal monotone.

We remark that, for single-valued maps $g$, the Yosida approximation can be used in (A3). If $g$ is the subdifferential of an indicatrix function, the inf-convolution of the indicatrix can be used to construct $g_{\delta}$ as in (A3).

Our homogenization result is based on the following existence result for the plasticity system. The proof is essentially as in [25]. Since we construct solutions with a high regularity (higher than energy estimates suggest), we can use a strong solution concept for system (1.2): The relation (1.2a) is satisfied in the sense of distributions, the relations $(1.2 \mathrm{~b})$ and $(1.2 \mathrm{c})$ are satisfied pointwise almost everywhere.

Theorem 2.1 (Existence). Let assumptions (A1)-(A3) on the coefficients be satisfied, let $f \in H^{1}\left(0, T ; L^{2}\left(\Omega ; \mathbb{R}^{n}\right)\right)$ be given, let initial data and boundary conditions of (2.1)(2.2) be given by $U_{0}^{\eta} \in H^{2}\left(0, T ; H^{1}\left(\Omega ; \mathbb{R}^{n}\right)\right) \cap H^{3}\left(0, T ; L^{2}\left(\Omega ; \mathbb{R}^{n}\right)\right), u_{0}^{\eta} \in H^{1}\left(\Omega ; \mathbb{R}^{n}\right), u_{1}^{\eta} \in$ 
$H^{1}\left(\Omega ; \mathbb{R}^{n}\right), \xi_{0}^{\eta} \in L^{2}\left(\Omega ; \mathbb{R}^{N}\right)$. We furthermore assume the compatibility $U_{0}^{\eta}(\cdot, 0)=u_{0}^{\eta}$ and, for $\sigma_{0}^{\eta}:=D_{\eta}\left(\nabla^{s} u_{0}^{\eta}-B_{\eta} \xi_{0}^{\eta}\right)$, that $B_{\eta}^{T} \sigma_{0}^{\eta}-L_{\eta} \xi_{0}^{\eta}$ maps into $K_{0}$. We assume the regularity $\nabla \cdot \sigma_{0}^{\eta} \in L^{2}\left(\Omega ; \mathbb{R}^{n}\right)$. Then there exists a unique strong solution $\left(u^{\eta}, \sigma^{\eta}, \xi^{\eta}\right)$ of problem (1.2). Furthermore, there exists a constant $c>0$ depending on $\Omega_{T}, D, L, B, \varrho, g$, but not on $\eta, f$, or the initial and boundary data, such that

$$
\begin{aligned}
& \quad\left\|u^{\eta}\right\|_{W^{1, \infty}\left(0, T ; H^{1}\left(\Omega ; \mathbb{R}^{n}\right)\right)}+\left\|\partial_{t}^{2} u^{\eta}\right\|_{L^{\infty}\left(0, T ; L^{2}\left(\Omega ; \mathbb{R}^{n}\right)\right)}+\left\|\sigma^{\eta}\right\|_{W^{1, \infty}\left(0, T ; L^{2}\left(\Omega ; \mathbb{R}_{s}^{n \times n}\right)\right)} \\
& +\left\|\xi^{\eta}\right\|_{W^{1, \infty}\left(0, T ; L^{2}\left(\Omega ; \mathbb{R}^{N}\right)\right)} \leq c\left(1+\left\|u_{0}^{\eta}\right\|_{H^{1}\left(\Omega ; \mathbb{R}^{n}\right)}+\left\|u_{1}^{\eta}\right\|_{H^{1}\left(\Omega ; \mathbb{R}^{n}\right)}+\left\|\xi_{0}^{\eta}\right\|_{L^{2}\left(\Omega ; \mathbb{R}^{N}\right)}\right. \\
& \left.+\left\|U_{0}^{\eta}\right\|_{H^{2}\left(0, T ; H^{1}\left(\Omega ; \mathbb{R}^{n}\right)\right) \cap H^{3}\left(0, T ; L^{2}\left(\Omega ; \mathbb{R}^{n}\right)\right)}+\left\|\nabla \cdot \sigma_{0}^{\eta}\right\|_{L^{2}\left(\Omega ; \mathbb{R}^{n}\right)}+\|f\|_{H^{1}\left(0, T ; L^{2}(\Omega)\right)}\right) .
\end{aligned}
$$

The strong solution concept of this existence result allows to multiply (1.2a) with $\partial_{t} u^{\eta}$ to obtain an energy balance relation. For $t$-independent boundary conditions $U_{0}^{\eta}$, the energy balance states: For almost every $t \in(0, T)$, there holds

$$
\begin{aligned}
\int_{\Omega_{t}} \varrho_{\eta} \partial_{t}^{2} u^{\eta} \cdot \partial_{t} u^{\eta}+ & \int_{\Omega_{t}} \partial_{t} \sigma^{\eta}: D_{\eta}^{-1} \sigma^{\eta}+\int_{\Omega_{t}} \partial_{t} \xi^{\eta} \cdot L_{\eta} \xi^{\eta}+\int_{\Omega_{t}} \partial_{t} \xi^{\eta} \cdot\left(B_{\eta}^{T} \sigma^{\eta}-L_{\eta} \xi^{\eta}\right) \\
& =\int_{\Omega_{t}} \partial_{t} u^{\eta} \cdot f .
\end{aligned}
$$

The proof of Theorem 2.1 is performed in Section 4. It is based on a Galerkin scheme and a regularization of the function $g$.

\section{Homogenized plasticity problem and main result}

Our main result is the homogenization of the plasticity system. We remark that assumption (A4) appears in the next section. The assumption is satisfied for subdifferentials $g$ and for piecewise $x$-independent operators $g$, see Lemmas 3.3 and 3.4.

Theorem 2.2 (Homogenization). Let the assumptions (A1)-(A4) be satisfied. For $\eta \rightarrow 0$, let $\left(u^{\eta}, \xi^{\eta}, \sigma^{\eta}\right)_{\eta}$ be a sequence of solutions to $(1.2)$ for $f \in H^{1}\left(0, T ; L^{2}\left(\Omega ; \mathbb{R}^{n}\right)\right)$ as in Theorem 2.1. Let the initial data satisfy

$$
\begin{aligned}
& u_{0}^{\eta} \rightarrow u_{0}, \quad u_{1}^{\eta} \rightarrow u_{1} \quad \text { strongly in } H^{1}(\Omega), \\
& \xi_{0}^{\eta} \stackrel{2}{\rightarrow} w_{0} \quad \text { strongly two-scale in } L^{2}(\Omega \times Y) .
\end{aligned}
$$

We assume that $U_{0}^{\eta}$ is bounded in $H^{2}\left(0, T ; H^{1}\left(\Omega ; \mathbb{R}^{n}\right)\right) \cap H^{3}\left(0, T ; L^{2}\left(\Omega ; \mathbb{R}^{n}\right)\right)$ and weakly convergent to $U_{0}$, and that $\sigma_{0}^{\eta}:=D_{\eta}\left(\nabla^{s} u_{0}^{\eta}-B_{\eta} \xi_{0}^{\eta}\right)$ has $\nabla \cdot \sigma_{0}^{\eta}$ bounded in $L^{2}(\Omega)$. Then, for functions

$$
\begin{aligned}
& u \in H^{2}\left(0, T ; L^{2}\left(\Omega ; \mathbb{R}^{n}\right)\right) \cap H^{1}\left(0, T ; H^{1}(\Omega)\right), \quad v \in L^{2}\left(\Omega_{T} ; H^{1}\left(Y ; \mathbb{R}^{n}\right)\right), \\
& w \in H^{1}\left(0, T ; L^{2}\left(\Omega \times Y ; \mathbb{R}^{N}\right)\right), \quad z \in H^{1}\left(0, T ; L^{2}\left(\Omega \times Y ; \mathbb{R}_{s}^{n \times n}\right)\right),
\end{aligned}
$$

the following convergences hold:

$$
\begin{aligned}
\partial_{t}^{2} u^{\eta} \rightarrow \partial_{t}^{2} u & \text { weakly in } L^{2}\left(\Omega_{T} ; \mathbb{R}^{n}\right), \\
u^{\eta} \rightarrow u \text { and } \partial_{t} u^{\eta} \rightarrow \partial_{t} u & \text { weakly in } H^{1}\left(\Omega_{T} ; \mathbb{R}^{n}\right), \\
\xi^{\eta} \stackrel{2}{\rightarrow} w \text { and } \partial_{t} \xi^{\eta} \stackrel{2}{\rightarrow} \partial_{t} w & \text { weakly two-scale in } L^{2}\left(\Omega_{T} \times Y\right), \\
\sigma^{\eta} \stackrel{2}{\rightarrow} z \text { and } \partial_{t} \sigma^{\eta} \stackrel{2}{\rightarrow} \partial_{t} z & \text { weakly two-scale in } L^{2}\left(\Omega_{T} \times Y\right), \\
\nabla^{s} u^{\eta} \stackrel{2}{\rightarrow} \nabla_{x}^{s} u+\nabla_{y}^{s} v & \text { weakly two-scale in } L^{2}\left(\Omega_{T} \times Y\right) .
\end{aligned}
$$


The limit $(u, v, w, z)$ is the unique strong solution of system (1.3) with initial and boundary conditions (2.3)-(2.5).

We note that also solutions to the limit system satisfy an energy balance relation. Due to the regularity of solutions, this relation can be obtained by testing the conservation law with $\partial_{t} u$. In the case of $t$-independent boundary data the energy balance reads: for almost every $t \in(0, T)$ there holds

$$
\int_{\Omega_{t}} \bar{\varrho} \partial_{t}^{2} u \cdot \partial_{t} u+\int_{\Omega_{t} \times Y}\left\{\partial_{t} z: D^{-1} z+\partial_{t} w \cdot L w+\partial_{t} w \cdot\left(B^{T} z-L w\right)\right\}=\int_{\Omega_{t}} \partial_{t} u \cdot f .
$$

\section{Two-scale homogenization and monotonicity}

This section is devoted to the proof of Theorem 2.2. A sequence $\left(u^{\eta}, \xi^{\eta}, \sigma^{\eta}\right)_{\eta}$ of solutions to the original problem is available by Theorem 2.1. The existence result provides additionally a priori estimates for solutions. These $\eta$-independent estimates guarantee, for appropriate limit functions $(u, v, w, z)$ as in (2.13)-(2.14) and a subsequence $\eta \rightarrow 0$ the convergences of (2.15)-(2.19). The appropriate compactness results for two-scale convergence are recalled in the next subsection.

To prove Theorem 2.2, the main step is to show that the limit $(u, v, w, z)$ is a solution of the limit system (1.3); we perform this part of the proof with a monotonicity argument in Subsection 3.3. The calculation will additionally provide the energy balance. Uniqueness for the limit system can be derived easily, we present the calculation in Subsection 3.4.

\subsection{Tools from two-scale convergence}

In this subsection we briefly review standard properties of two-scale convergence [4, 22], and some more recent results regarding functionals $[27,30]$. These properties will allow to perform the proof of Theorem 2.2.

Let $\left(u_{\eta}\right)_{\eta}$ be bounded sequence in $L^{2}\left(\Omega ; \mathbb{R}^{m}\right)$. The sequence $\left(u_{\eta}\right)_{\eta}$ is said to be weakly two-scale convergent to $u \in L^{2}\left(\Omega \times Y ; \mathbb{R}^{m}\right)$ iff

$$
\lim _{\eta \rightarrow 0} \int_{\Omega} u_{\eta}(x) \cdot \psi\left(x, \frac{x}{\eta}\right) d x=\int_{\Omega \times Y} u(x, y) \cdot \psi(x, y) d x d y
$$

holds for all admissible test-functions $\psi: \Omega \times Y \rightarrow \mathbb{R}^{m}$. In this case, we write $u_{\eta} \stackrel{2}{\rightarrow} u$. A function $\psi \in L^{2}\left(\Omega \times Y ; \mathbb{R}^{m}\right)$ is an admissible test-function iff

$$
\lim _{\eta \rightarrow 0} \int_{\Omega}\left|\psi\left(x, \frac{x}{\eta}\right)\right|^{2} d x=\int_{\Omega \times Y}|\psi(x, y)|^{2} d x d y .
$$

Functions $\psi$ that are continuous in one variable (or piecewise constant in one variable) are admissible. A sequence $u_{\eta} \stackrel{2}{\rightarrow} u$ is called strongly two-scale convergent (and we write $\left.u_{\eta} \stackrel{2}{\rightarrow} u\right)$ iff $\lim _{\eta \rightarrow 0}\left\|u_{\eta}\right\|_{L^{2}(\Omega)}=\|u\|_{L^{2}(\Omega \times Y)}$.

We extend the definitions to time-dependent functions by regarding time as a parameter (i.e. we do not resolve oscillations in $t$ ): For a space-time cylinder $\Omega_{T}:=\Omega \times$ 
$(0, T)$ we call $\left(u_{\eta}\right)_{\eta}$ in $L^{2}\left(\Omega_{T} ; \mathbb{R}^{m}\right)$ weakly two-scale convergent to $u \in L^{2}\left(\Omega_{T} \times Y ; \mathbb{R}^{m}\right)$ iff

$$
\lim _{\eta \rightarrow 0} \int_{\Omega_{T}} u_{\eta}(x, t) \cdot \psi\left(x, \frac{x}{\eta}, t\right) d x d t=\int_{\Omega_{T} \times Y} u(x, y, t) \cdot \psi(x, y, t) d x d y d t
$$

for all $\psi(x, y, t)=\psi_{0}(x, y) \vartheta(t)$ with $\vartheta \in \mathcal{D}((0, T) ; \mathbb{R})$ and $\psi_{0}$ admissible. Strong twoscale convergence is defined as in the time-independent case.

The first two statements of the subsequent lemma recall some standard compactness results and limit characterizations from two-scale convergence theory; they can be found in [4]. The third statement concerning the matrix-valued case is a straightforward extension and was also used, e.g., in [27].

Lemma 3.1 (Tools from two-scale convergence). For any bounded sequence $\left(u_{\eta}\right)_{\eta}$ in $L^{2}\left(\Omega ; \mathbb{R}^{n}\right)$ there exists $u \in L^{2}\left(\Omega \times Y ; \mathbb{R}^{n}\right)$ such that, possibly extracting a subsequence,

$$
u_{\eta} \stackrel{2}{\rightarrow} u \quad \text { in } L^{2}\left(\Omega \times Y ; \mathbb{R}^{n}\right) .
$$

Let $\left(u_{\eta}\right)_{\eta}$ be a weakly convergent sequence, $u_{\eta} \rightarrow u$ in $H^{1}\left(\Omega ; \mathbb{R}^{n}\right)$. Then, for a subsequence and some $v \in L^{2}\left(\Omega ; H^{1}\left(Y ; \mathbb{R}^{n}\right)\right)$ with $\int_{Y} v(\cdot, y) d y=0$ there holds

$$
\nabla^{s} u_{\eta} \stackrel{2}{\rightarrow} \nabla^{s} u+\nabla_{y}^{s} v \quad \text { in } L^{2}\left(\Omega \times Y ; \mathbb{R}_{s}^{n \times n}\right) .
$$

Let $\left(\sigma_{\eta}\right)_{\eta}$ be a bounded sequence in $L^{2}\left(\Omega ; \mathbb{R}_{s}^{n \times n}\right)$ such that $\eta \nabla \cdot \sigma_{\eta}$ is bounded in $L^{2}\left(\Omega ; \mathbb{R}^{n}\right)$. Then, for a subsequence and some $\sigma \in L^{2}\left(\Omega \times Y ; \mathbb{R}_{s}^{n \times n}\right)$ with $\nabla_{x} \cdot \sigma \in$ $L^{2}\left(\Omega \times Y ; \mathbb{R}^{n}\right)$ there holds

$$
\sigma_{\eta} \stackrel{2}{\rightarrow} \sigma \quad \text { in } L^{2}\left(\Omega \times Y ; \mathbb{R}_{s}^{n \times n}\right), \quad \eta \nabla \cdot \sigma_{\eta} \stackrel{2}{\rightarrow} \nabla_{y} \cdot \sigma \quad \text { in } L^{2}\left(\Omega \times Y ; \mathbb{R}^{n}\right) .
$$

In particular, if $\nabla \cdot \sigma_{\eta}$ is bounded in $L^{2}\left(\Omega ; \mathbb{R}^{n}\right)$, then $\nabla_{y} \cdot \sigma=0$.

All these results extend to the time-dependent case.

We note that the time-dependent case can be treated by using $\Omega_{T}$ as the basic domain; the two-scale limits can be averaged over the fast time-scale, since we use only slowly varying test-functions $\vartheta$ in the time variable.

In addition to the above properties, we need the lower semi-continuity of convex functionals. This property was derived in [30], Proposition 1.3. We use the Borel $\sigma$-algebras $\mathcal{B}\left(\mathbb{R}^{M}\right), \mathcal{B}(\Omega), \mathcal{B}(Y)$ and the Lebesgue $\sigma$-algebras $\mathcal{L}\left(\mathbb{R}^{M}\right), \mathcal{L}(\Omega), \mathcal{L}(Y)$. We remark that the measurability assumptions on $\Psi$ are satisfied for Caratheodory functions (compare [7], page 30).

Proposition 3.2 (Visintin 2007, Two-scale limits in nonlinear functionals). Let $\Psi$ : $\mathbb{R}^{m} \times \Omega \times Y \rightarrow \mathbb{R} \cup\{+\infty\}$ be measurable either w.r.t. $\mathcal{B}\left(\mathbb{R}^{m}\right) \otimes \mathcal{B}(\Omega) \otimes \mathcal{L}(Y)$ or w.r.t. $\mathcal{B}\left(\mathbb{R}^{m}\right) \otimes \mathcal{L}(\Omega) \otimes \mathcal{B}(Y)$. Let $u_{\eta} \stackrel{2}{\rightarrow} u$ in $L^{2}\left(\Omega \times Y ; \mathbb{R}^{m}\right)$ be a weakly two-scale convergent sequence.

1. Assume that $\Psi$ has the following properties: (i) $\Psi(v, x, y) \geq 0$ for all $v$, for a.e. $(x, y)$, (ii) the function $v \mapsto \Psi(v, x, y)$ is convex for a.e. $(x, y)$, (iii) the function $(v, x) \mapsto \Psi(v, x, y)$ is lower semicontinuous for a.e. $y$. Then

$$
\liminf _{\eta \rightarrow 0} \int_{\Omega} \Psi\left(u_{\eta}(x), x, \frac{x}{\eta}\right) d x \geq \int_{\Omega \times Y} \Psi(u(x, y), x, y) d x d y .
$$


2. Assume that $(v, x) \mapsto \Psi(v, x, y)$ is continuous for a.e. $y$ and that there exists $c>0$ such that $|\Psi(v, x, y)| \leq c|v|^{2}$ for a.e. $(x, y)$. Then, if $u_{\eta} \stackrel{2}{\rightarrow} u$ in $L^{2}\left(\Omega \times Y ; \mathbb{R}^{m}\right)$ is strongly two-scale convergent, there holds

$$
\lim _{\eta \rightarrow 0} \int_{\Omega} \Psi\left(u_{\eta}(x), x, \frac{x}{\eta}\right) d x=\int_{\Omega \times Y} \Psi(u(x, y), x, y) d x d y .
$$

\subsection{A property of the flow function $g$}

In our homogenization theorem we assume that the map $g: \mathbb{R}^{N} \times \Omega \times Y \rightarrow \mathcal{P}\left(\mathbb{R}^{N}\right)$ (the map $g(\cdot ; x, y)$ is multi-valued from $\mathbb{R}^{N}$ to $\mathbb{R}^{N}$ ) has the subsequent property (A4). We will show below that a regularity assumption on the maximal monotone map $g$ is sufficient to obtain property (A4).

(A4) For weakly two-scale convergent sequences $f^{\eta} \stackrel{2}{\rightarrow} f$ and $h^{\eta} \stackrel{2}{\rightarrow} h$ in $L^{2}\left(\Omega_{T} \times Y ; \mathbb{R}^{N}\right)$ with $h^{\eta}(x, t) \in g\left(f^{\eta}(x, t) ; x, x / \eta\right)$, the product lim-inf inequality

$$
\liminf _{\eta \rightarrow 0} \int_{\Omega_{T}} h^{\eta} \cdot f^{\eta} \leq \int_{\Omega_{T} \times Y} h \cdot f
$$

implies $h(x, y, t) \in g(f(x, y, t) ; x, y)$.

Our first observation is that (A4) is satisfied for subdifferentials $g$. We note that we could, alternatively, conclude (A4) from Theorem 2.1 in [30] (under slightly different assumptions on $\Psi$, in particular, without lower semi-continuity).

Lemma 3.3 (Assumption (A4) for subdifferentials). Let $g$ be a subdifferential of $\Psi$ : $\mathbb{R}^{N} \times \Omega \times Y \rightarrow \mathbb{R} \cup\{\infty\}$, where $\Psi$ has the measurability properties of Proposition 3.2, is non-negative, convex, and lower semi-continuous. Then $g(\cdot ; x, y)=\partial \Psi(\cdot ; x, y)$ satisfies (A4).

Proof. We calculate with sequences $f^{\eta}$ and $h^{\eta}$ as in (A4). We use in (i) the definition of the conjugate function $\Psi^{*}$, in (ii) the lower semi-continuity property of Proposition 3.2 , in (iii) the Fenchel characterization of the property $h^{\eta}(x, t) \in \partial \Psi_{\eta}\left(f^{\eta}(x, t) ; x, x / \eta\right)$, and in (iv) the assumption (3.3):

$$
\begin{aligned}
\int_{\Omega_{T} \times Y} h \cdot f & \stackrel{(i)}{\leq} \int_{\Omega_{T} \times Y} \Psi(f)+\Psi^{*}(h) \stackrel{(i i)}{\leq} \liminf _{\eta \rightarrow 0} \int_{\Omega_{T}} \Psi_{\eta}\left(f^{\eta}\right)+\Psi_{\eta}^{*}\left(h^{\eta}\right) \\
& \stackrel{(i i i)}{=} \liminf _{\eta \rightarrow 0} \int_{\Omega_{T}} f^{\eta} \cdot h^{\eta} \stackrel{(i v)}{\leq} \int_{\Omega_{T} \times Y} h \cdot f .
\end{aligned}
$$

We conclude that all inequalities are equalities. Equality of the first two integrals implies, due to the pointwise inequality, that the integrands coincide almost everywhere. This, together with the Fenchel characterization of the subdifferential, provides $h \in g(f)$.

The next lemma states that general maximal monotone maps $g$ satisfy (A4) under some regularity assumption. We provide a statement for piecewise $x$-independent maps $g$, we expect that it is possible to replace this condition by a continuity property in $x$, as in Theorem 3.1 in [30]. Nevertheless, since in our application unbounded maps $g$ are relevant, we cannot use the result of [30]. 
We call a map $G: \Omega \rightarrow Z$ piecewise constant in $x$ if, for a finite number of disjoint open subsets $\left(\Omega_{j}\right)_{j=1, \ldots, J}$ with $\left|\Omega \backslash \bigcup_{j} \Omega_{j}\right|=0$ and $J$ elements $G_{j} \in Z$, there holds $G(x)=G_{j}$ for every $x \in \Omega_{j}$.

Lemma 3.4 (Assumption (A4) for piecewise $x$-independent functions). Let $g(\cdot ; x, y)$ : $\mathbb{R}^{N} \rightarrow \mathcal{P}\left(\mathbb{R}^{N}\right)$ be as in (A2), maximal monotone and measurable. If $g$ is piecewise constant in $x$, then (A4) is satisfied.

Proof. Let $f^{\eta}(x, t), h^{\eta}(x, t)$ with limits $f(x, y, t), h(x, y, t)$ be sequences as in (A4). We suppress the parameter $t$ in the following.

Step 1: Maximal monotonicity and admissibility issues. In this step of the proof we consider two arbitrary functions $f_{0}, h_{0}: \Omega_{T} \times Y \rightarrow \mathbb{R}^{N}$ of class $L^{2}\left(\Omega_{T} \times Y\right)$ that satisfy $h_{0} \in g\left(f_{0}\right)$, and assume that both functions are admissible test-functions in the sense of two-scale convergence. For the corresponding oscillatory functions $f_{0, \eta}(x, t)=$ $f_{0}(x, x / \eta, t)$ and $h_{0, \eta}(x, t)=h_{0}(x, x / \eta, t)$ we observe that, due to monotonicity of $g$,

$$
0 \leq \int_{\Omega_{T}}\left(h_{0, \eta}-h^{\eta}\right) \cdot\left(f_{0, \eta}-f^{\eta}\right) .
$$

We take the limes inferior $\eta \rightarrow 0$, exploiting the definition of two-scale convergence and the property (3.3) of the product $h^{\eta} \cdot f^{\eta}$ to obtain

$$
0 \leq \int_{\Omega_{T} \times Y}\left(h_{0}-h\right) \cdot\left(f_{0}-f\right) .
$$

The multi-valued operator $g(\cdot ; x, y)$ defines a multi-valued operator on the function space, $g_{\Omega_{T} \times Y}: L^{2}\left(\Omega_{T} \times Y\right) \rightarrow \mathcal{P}\left(L^{2}\left(\Omega_{T} \times Y\right)\right)$. At first sight, it seems that (3.4) provides $h \in g(f)$ by maximal monotonicity of $g_{\Omega_{T} \times Y}$. But there is an issue regarding the admissibility of the test-functions: Since (3.4) holds only for admissible $h_{0}$ and $f_{0}$, we cannot conclude $h \in g(f)$ from maximality of $g_{\Omega_{T} \times Y}$.

Step 2: Proof for piecewise $x$-independent $g$. Let $\left(\Omega_{j}\right)_{j=1, \ldots, J}$ be open subsets such that $g$ does not depend on $x$ in $\Omega_{j}$. We claim that, for almost every point $\left(x_{0}, t_{0}\right) \in$ $\bigcup_{j} \Omega_{j} \times(0, T)$ and arbitrary $F_{0}, H_{0} \in L^{2}\left(Y ; \mathbb{R}^{N}\right)$ with $H_{0}(\cdot) \in g\left(F_{0}(\cdot), x_{0}, \cdot\right)$, there holds

$$
0 \leq \int_{Y}\left(H_{0}(y)-h\left(x_{0}, y, t_{0}\right)\right) \cdot\left(F_{0}(y)-f\left(x_{0}, y, t_{0}\right)\right) d y .
$$

Once (3.5) is verified, the maximality of the multi-valued maximal monotone operator $g_{Y}\left(\cdot ; x_{0}, \cdot\right): L^{2}(Y) \rightarrow L^{2}(Y)$ implies $h\left(x_{0}, \cdot, t_{0}\right) \in g\left(f\left(x_{0}, \cdot, t_{0}\right) ; x_{0}, \cdot\right)$ almost everywhere in $Y$. This is the result, since $\left(x_{0}, t_{0}\right)$ was arbitrary in a set of full measure.

In order to show (3.5), let $j \leq J$ be an index and $F_{0}, H_{0} \in L^{2}\left(Y, \mathbb{R}^{N}\right)$ be such that $H_{0}(\cdot) \in g\left(F_{0}(\cdot) ; x, \cdot\right)$ for every $x \in \Omega_{j}$. We can assume that the arbitrary point $\left(x_{0}, t_{0}\right) \in \Omega_{j} \times(0, T)$ is a Lebesgue point for

$$
\Omega_{j} \times(0, T) \ni(x, t) \mapsto \int_{Y}\left(H_{0}(y)-h(x, y, t)\right) \cdot\left(F_{0}(y)-f(x, y, t)\right) d y .
$$

For a small radius $r>0$, using the characteristic function $\mathbf{1}_{B}$ of a ball $B \subset \Omega_{T}$, we set $h_{0}(x, y, t):=H_{0}(y) \mathbf{1}_{B_{r}\left(x_{0}, t_{0}\right)}(x, t)$ and $f_{0}(x, y, t):=F_{0}(y) \mathbf{1}_{B_{r}\left(x_{0}, t_{0}\right)}(x, t)$. These two functions are admissible in the sense of two-scale convergence since they are piecewise constant in one variable (regarding $(x, t) \in \Omega_{T}$ as one variable). Furthermore, they 
satisfy $h_{0}(x, y, t) \in g\left(f_{0}(x, y, t) ; x, y\right)$ (we recall that we introduced the normalization $0 \in g(0 ; x, y))$. Since the functions are admissible, inequality (3.4) is satisfied. We divide by the volume of the ball $B_{r}=B_{r}\left(x_{0}, t_{0}\right)$ and take the limit $r \searrow 0$ to obtain

$$
0 \leq \liminf _{r \rightarrow 0} \frac{1}{\left|B_{r}\right|} \int_{B_{r}} \int_{Y}\left(h_{0}-h\right) \cdot\left(f_{0}-f\right)=\int_{Y}\left(H_{0}-h\left(x_{0}, \cdot, t_{0}\right)\right) \cdot\left(F_{0}-f\left(x_{0}, \cdot, t_{0}\right)\right) .
$$

Since $\left(x_{0}, t_{0}\right)$ was arbitrary, we obtain the claim of (3.5).

\subsection{Proof of the homogenization result}

We now perform the rigorous derivation of the homogenization result. Our approach uses the energy balance for the $\eta$-problem and exploits the lower semi-continuity of the energies in the limit $\eta \rightarrow 0$. For ease of notation we consider only time-independent boundary data $U_{0}^{\eta}$ in this proof. The general case can be concluded along the same lines.

Proof of Theorem 2.2. In the first paragraph of Section 3, we observed that the bounds (2.10) allow to choose a subsequence $\eta \rightarrow 0$ and limit functions $(u, v, w, z)$ with the regularity (2.13)-(2.14) such that the convergences (2.15)-(2.19) hold. We exploit here that norms of $\nabla^{s} u^{\eta}$ are controlled by the corresponding norms of $\sigma^{\eta}$ and $\xi^{\eta}$ through (1.2b).

The initial and boundary conditions $(2.3)-(2.5)$ of the limit functions are satisfied due to the existence of traces of the corresponding functions and (2.12).

It remains to show that the limit $(u, v, w, z)$ solves the limit problem (1.3), and the uniqueness of solutions.

Step 1: The linear relations (1.3a)-(1.3c). In order to prove (1.3a), it is sufficient to use a (single-scale) smooth test-function in (1.2a). We multiply with $\varphi \in \mathcal{D}\left(\Omega_{T} ; \mathbb{R}^{n}\right)$, integrate over $\Omega_{T}$ and pass to the limit $\eta \rightarrow 0$. Since weighted time integrals of $\partial_{t} u^{\eta}$ converge strongly in $L^{2}(\Omega)$ and $\varrho_{\eta}$ converges weakly in $L^{2}(\Omega)$, we obtain

$$
\begin{aligned}
\lim _{\eta \rightarrow 0} & \int_{\Omega_{T}} \varrho\left(x, \frac{x}{\eta}\right) \partial_{t}^{2} u^{\eta}(x, t) \cdot \varphi(x, t) d x d t \\
& =-\lim _{\eta \rightarrow 0} \int_{\Omega_{T}} \varrho_{\eta}(x) \partial_{t} u^{\eta}(x, t) \cdot \partial_{t} \varphi(x, t) d x d t \\
& =-\int_{\Omega_{T}} \bar{\varrho}(x) \partial_{t} u(x, t) \cdot \partial_{t} \varphi(x, t) d x d t=\int_{\Omega_{T}} \bar{\varrho}(x) \partial_{t}^{2} u(x, t) \cdot \varphi(x, t) d x d t .
\end{aligned}
$$

For the other term in $(1.2 \mathrm{a})$ we find

$$
\begin{aligned}
& \lim _{\eta \rightarrow 0} \int_{\Omega_{T}} \nabla \cdot \sigma^{\eta}(x, t) \cdot \varphi(x, t) d x d t=-\lim _{\eta \rightarrow 0} \int_{\Omega_{T}} \sigma^{\eta}(x, t): \nabla \varphi(x, t) d x d t \\
& \quad=-\int_{\Omega_{T} \times Y} z(x, y, t): \nabla \varphi(x, t) d x d t=\int_{\Omega_{T}} \nabla \cdot\left(f_{Y} z(x, y, t) d y\right) \cdot \varphi(x, t) d x d t .
\end{aligned}
$$

Taking the limit in the integral containing $f$ does not pose any difficulty and we obtain (1.3a) in the sense of distributions. 
We next derive (1.3b) from (1.2b), using the definition of two-scale convergence. We use an arbitrary test-function $\psi \in \mathcal{D}\left(\Omega_{T} \times Y ; \mathbb{R}_{s}^{n \times n}\right)$ and obtain for the stress variable

$$
\lim _{\eta \rightarrow 0} \int_{\Omega_{T}} \sigma^{\eta}(x, t): \psi\left(x, \frac{x}{\eta}, t\right) d x d t=\int_{\Omega_{T} \times Y} z(x, y, t): \psi(x, y, t) d y d x d t .
$$

On the other side of $(1.2 \mathrm{~b})$, two similar terms appear. We use the abbreviations $\psi_{1}(x, y, t):=D^{T}(x, y) \psi(x, y, t)$ and $\psi_{2}(x, y, t):=B^{T}(x, y) D^{T}(x, y) \psi(x, y, t)$. With Lemma 3.1 and the fact that the product $B^{T} D^{T}$ is again an admissible test-function we obtain from (2.17) and (2.19)

$$
\begin{aligned}
\lim _{\eta \rightarrow 0} & \int_{\Omega_{T}} D\left(x, \frac{x}{\eta}\right)\left(\nabla^{s} u^{\eta}(x, t)-B\left(x, \frac{x}{\eta}\right) \xi^{\eta}(x, t)\right): \psi\left(x, \frac{x}{\eta}, t\right) d x d t \\
= & \lim _{\eta \rightarrow 0} \int_{\Omega_{T}} \nabla^{s} u^{\eta}(x, t): \psi_{1}\left(x, \frac{x}{\eta}, t\right)-\xi^{\eta}(x, t): \psi_{2}\left(x, \frac{x}{\eta}, t\right) d x d t \\
= & \int_{\Omega_{T} \times Y}\left(\nabla^{s} u(x, t)+\nabla_{y}^{s} v(x, y, t)\right): \psi_{1}(x, y, t) d y d x d t \\
& -\int_{\Omega_{T} \times Y} w(x, y, t): \psi_{2}(x, y, t) d y d x d t \\
= & \int_{\Omega_{T} \times Y} D(x, y)\left(\nabla^{s} u^{\eta}(x, t)+\nabla_{y}^{s} v(x, y, t)-B(x, y) w(x, y, t)\right): \psi(x, y, t) d y d x d t .
\end{aligned}
$$

Since $\psi$ was arbitrary, we obtain (1.3b). Equation (1.3c) follows directly from the boundedness of $\nabla \cdot \sigma^{\eta}$ in $L^{2}\left(\Omega_{T}\right)$, see the last conclusion in Lemma 3.1.

Step 2: A product of weakly convergent sequences. Our next aim is to verify the (nonlinear) flow-rule (1.3d). To this end, we show an inequality regarding the limit of a certain product. We claim that, for almost every $t \in(0, T)$,

$$
\limsup _{\eta \rightarrow 0} \int_{\Omega_{t}} \partial_{t} \xi^{\eta} \cdot\left(B_{\eta}^{T} \sigma^{\eta}-L_{\eta} \xi^{\eta}\right) \leq \int_{\Omega_{t} \times Y} \partial_{t} w \cdot\left(B^{T} z-L w\right) .
$$

We will obtain (3.6) from the energy balance (2.11) and the lower semi-continuity result of Proposition 3.2. We fix $t \in(0, T)$ such that the energy balance holds for all $\eta$ of the sequence $\eta \rightarrow 0$. Additionally, we demand that $t$ is a Lebesgue point for the functions $w$ and $z$. We write (2.11) as

$$
\begin{aligned}
& \int_{\Omega_{t}} \partial_{t} \xi^{\eta} \cdot\left(B_{\eta}^{T} \sigma^{\eta}-L_{\eta} \xi^{\eta}\right) \\
& \quad=\int_{\Omega_{t}} \partial_{t} u^{\eta} \cdot f-\int_{\Omega_{t}} \varrho_{\eta} \partial_{t}^{2} u^{\eta} \cdot \partial_{t} u^{\eta}-\int_{\Omega_{t}} \partial_{t} \sigma^{\eta}: D_{\eta}^{-1} \sigma^{\eta}-\int_{\Omega_{t}} \partial_{t} \xi^{\eta} \cdot L_{\eta} \xi^{\eta} \\
& \quad=: I_{1}^{\eta}-I_{2}^{\eta}-I_{3}^{\eta}-I_{4}^{\eta} .
\end{aligned}
$$

In the integral $I_{1}^{\eta}$, the limit can be obtained directly from the convergence $(2.16)$,

$$
\lim _{\eta \rightarrow 0} I_{1}^{\eta}=\lim _{\eta \rightarrow 0} \int_{\Omega_{t}} \partial_{t} u^{\eta} \cdot f=\int_{\Omega_{t}} \partial_{t} u \cdot f .
$$

The other three integrals are similar in their structure; we treat here only one term, for notational convenience we choose the integral $I_{4}^{\eta}$. We note first that the two-scale 
convergence $\xi^{\eta} \stackrel{2}{\rightarrow} w$ on $\Omega_{T} \times Y$ implies, due to the additional time regularity of $\xi^{\eta}$ and $w$, the inequality

$$
\liminf _{\eta \rightarrow 0} \int_{\Omega} \xi^{\eta}(x, t) \cdot L_{\eta}(x) \xi^{\eta}(x, t) d x \geq \int_{\Omega \times Y} w(x, y, t) \cdot L(x, y) w(x, y, t) d x d y .
$$

Indeed, since $\partial_{t} \xi^{\eta} \in L^{2}\left(0, T ; L^{2}(\Omega)\right)$ is uniformly bounded, we find for arbitrary $\gamma<$ $1 / 2$ a uniform bound for $\xi^{\eta} \in C^{\gamma}\left(0, T ; L^{2}(\Omega)\right)$. In particular, for every $\delta>0$ and an error term $q=q(\delta)$ with $\lim _{\delta \rightarrow 0} q(\delta)=0$, there holds

$$
\begin{aligned}
\liminf _{\eta \rightarrow 0} & \int_{\Omega} \xi^{\eta}(x, t) \cdot L_{\eta}(x) \xi^{\eta}(x, t) d x \\
& \geq \liminf _{\eta \rightarrow 0} f_{0}^{\delta} \int_{\Omega} \xi^{\eta}(x, t+\tau) \cdot L_{\eta}(x) \xi^{\eta}(x, t+\tau) d x d \tau+q(\delta) \\
& \geq f_{0}^{\delta} \int_{\Omega \times Y} w(x, y, t+\tau) \cdot L(x, y) w(x, y, t+\tau) d x d y d \tau+q(\delta) .
\end{aligned}
$$

In the second inequality, we exploited the lower semi-continuity result of Proposition 3.2. Taking the limit $\delta \rightarrow 0$, since $t$ is a Lebesgue point of $w$, we obtain the claim of (3.9).

The function $\xi^{\eta} \in H^{1}\left(0, T ; L^{2}(\Omega)\right)$ has a continuous representative for which we can calculate, using additionally the strong two-scale convergence of $\xi_{0}^{\eta}$ of (2.12) and Proposition 3.2

$$
\begin{aligned}
\limsup _{\eta \rightarrow 0}\left(-I_{4}^{\eta}\right) & =-\liminf _{\eta \rightarrow 0} \int_{0}^{t} \int_{\Omega} \partial_{t} \xi^{\eta} \cdot L_{\eta} \xi^{\eta} \\
& =-\liminf _{\eta \rightarrow 0}\left[\frac{1}{2} \int_{\Omega} \xi^{\eta}(t) \cdot L_{\eta} \xi^{\eta}(t)-\frac{1}{2} \int_{\Omega} \xi_{0}^{\eta} \cdot L_{\eta} \xi_{0}^{\eta}\right] \\
& \stackrel{(3.9)}{\leq}-\frac{1}{2} \int_{\Omega \times Y} w(t) \cdot L w(t)+\frac{1}{2} \int_{\Omega \times Y} w_{0} \cdot L w_{0}=-\int_{\Omega_{t} \times Y} \partial_{t} w \cdot L w
\end{aligned}
$$

The integrals $I_{2}^{\eta}$ and $I_{3}^{\eta}$ are analyzed in a similar way: we use Proposition 3.2, with $\Psi(v, x, y):=\varrho(x, y) v \cdot v$ to treat $I_{2}^{\eta}$, and with $\Psi(v, x, y):=v \cdot D^{-1}(x, y) v$ to treat $I_{3}^{\eta}$. As above, we obtain

$$
\begin{aligned}
& \limsup _{\eta \rightarrow 0}\left(-I_{2}^{\eta}\right) \leq-\int_{\Omega_{t} \times Y} \varrho \partial_{t}^{2} u \cdot \partial_{t} u=-\int_{\Omega_{t}} \bar{\varrho} \partial_{t}^{2} u \cdot \partial_{t} u, \\
& \limsup _{\eta \rightarrow 0}\left(-I_{3}^{\eta}\right) \leq-\int_{\Omega_{t} \times Y} \partial_{t} z: D^{-1} z .
\end{aligned}
$$

After this preparation, we take the limes superior in (3.7) and obtain

$$
\begin{aligned}
\limsup _{\eta \rightarrow 0} & \int_{\Omega_{t}} \partial_{t} \xi^{\eta} \cdot\left(B_{\eta}^{T} \sigma^{\eta}-L_{\eta} \xi^{\eta}\right) \\
& \leq \limsup _{\eta \rightarrow 0}\left(I_{1}^{\eta}\right)+\underset{\eta \rightarrow 0}{\limsup }\left(-I_{2}^{\eta}\right)+\underset{\eta \rightarrow 0}{\limsup }\left(-I_{3}^{\eta}\right)+\underset{\eta \rightarrow 0}{\limsup }\left(-I_{4}^{\eta}\right) \\
& \leq \int_{\Omega_{t}} \partial_{t} u \cdot f-\int_{\Omega_{t}} \bar{\varrho} \partial_{t}^{2} u \cdot \partial_{t} u-\int_{\Omega_{t} \times Y} \partial_{t} z: D^{-1} z-\int_{\Omega_{t} \times Y} \partial_{t} w \cdot L w \\
& \stackrel{(1.3 \mathrm{a})}{=}-\int_{\Omega_{t}} \partial_{t} u \cdot(\nabla \cdot \bar{z})-\int_{\Omega_{t} \times Y} \partial_{t} z: D^{-1} z-\int_{\Omega_{t} \times Y} \partial_{t} w \cdot L w
\end{aligned}
$$




$$
\begin{aligned}
& =\int_{\Omega_{t} \times Y} \partial_{t} \nabla^{s} u: z-\int_{\Omega_{t} \times Y} \partial_{t} z: D^{-1} z-\int_{\Omega_{t} \times Y} \partial_{t} w \cdot L w \\
& =\int_{\Omega_{t} \times Y} \partial_{t}\left(\nabla^{s} u-D^{-1} z\right): z-\int_{\Omega_{t} \times Y} \partial_{t} w \cdot L w \\
& \stackrel{(1.3 \mathrm{~b})}{=} \int_{\Omega_{t} \times Y} \partial_{t}\left(B w-\nabla_{y} v\right): z-\int_{\Omega_{t} \times Y} \partial_{t} w \cdot L w \\
& \stackrel{(1.3 \mathrm{c})}{=} \int_{\Omega_{t} \times Y} \partial_{t} w \cdot\left(B^{T} z-L w\right) .
\end{aligned}
$$

We have thus shown (3.6).

We observe that the last computation contains also the energy balance relation (2.20) for the limit system.

Step 3: The flow-rule. The nonlinear relation (1.3d) can now be concluded from (3.6) using property (A4) of the monotone flow function $g$. We use

$$
f^{\eta}:=B_{\eta}^{T} \sigma^{\eta}-L_{\eta} \xi^{\eta}, \quad h^{\eta}:=\partial_{t} \xi^{\eta} .
$$

The flow rule is satisfied on the $\eta$-level, hence $h^{\eta} \in g_{\eta}\left(f^{\eta}\right)$. The (weak) two-scale limit functions are $f(x, y, t):=B(x, y)^{T} z(x, y, t)-L(x, y) w(x, y, t)$ and $h(x, y, t):=$ $\partial_{t} w(x, y, t)$. With these definitions, inequality (3.6) implies (3.3), hence all prerequisites of (A4) are satisfied. Property (A4) provides $h(x, y, t) \in g(f(x, y, t) ; x, y)$, which is nothing but the flow rule $(1.3 \mathrm{~d})$.

With the observation of uniqueness for the limit system (see the next Subsection), the proof of Theorem 2.2 is complete.

\subsection{Uniqueness for the limit system}

In order to conclude Theorem 2.2, we have to show uniqueness of the solution $(u, v, w, z)$ of the limit system (this implies, in particular, the convergence of the original sequence). In order to show uniqueness, we consider two strong solutions $\left(u_{i}, v_{i}, w_{i}, z_{i}\right)$ for $i \in\{1,2\}$ of system (1.3) with identical initial and boundary conditions (2.3)(2.5). We define $(\tilde{u}, \tilde{v}, \tilde{w}, \tilde{z}):=\left(u_{2}, v_{2}, w_{2}, z_{2}\right)-\left(u_{1}, v_{1}, w_{1}, z_{1}\right)$ as the difference of the two solutions. We compute

$$
\begin{aligned}
-\int_{\Omega} \bar{\varrho} \partial_{t}^{2} \tilde{u} \cdot \partial_{t} \tilde{u} & \stackrel{(1.3 \mathrm{a})}{=} \int_{\Omega}\left(f_{Y} \tilde{z}\right): \nabla^{s} \partial_{t} \tilde{u}=\int_{\Omega \times Y} \tilde{z}: \partial_{t} \nabla^{s} \tilde{u} \\
& \stackrel{(1.3 \mathrm{~b})}{=} \int_{\Omega \times Y} \tilde{z}: \partial_{t}\left(D^{-1} \tilde{z}+B \tilde{w}-\nabla_{y}^{s} \tilde{v}\right) \stackrel{(1.3 \mathrm{c})}{=} \int_{\Omega \times Y} \tilde{z}: \partial_{t}\left(D^{-1} \tilde{z}+B \tilde{w}\right) \\
& =\int_{\Omega \times Y} D^{-1} \tilde{z}: \partial_{t} \tilde{z}+\left(B^{T} \tilde{z}-L \tilde{w}+L \tilde{w}\right): \partial_{t} \tilde{w} .
\end{aligned}
$$

Since the initial data are identical, integrating over time from 0 to $t$ and exploiting the monotonicity (2.9) of $g$ we obtain

$$
\begin{aligned}
-\frac{1}{2} \int_{\Omega} \bar{\varrho}\left|\partial_{t} \tilde{u}(t)\right|^{2} & -\frac{1}{2} \int_{\Omega \times Y} D^{-1} \tilde{z}(t): \tilde{z}(t)-\frac{1}{2} \int_{\Omega \times Y} L \tilde{w}(t): \tilde{w}(t) \\
& =\int_{\Omega_{t} \times Y}\left(\left(B^{T} z_{2}-L w_{2}\right)-\left(B^{T} z_{1}-L w_{1}\right)\right):\left(\partial_{t} w_{2}-\partial_{t} w_{1}\right) \stackrel{(1.3 \mathrm{~d})}{\geq} 0 .
\end{aligned}
$$

From positivity of $\bar{\varrho}, D^{-1}$, and $L$, we conclude $\left(u_{2}, v_{2}, w_{2}, z_{2}\right)=\left(u_{1}, v_{1}, w_{1}, z_{1}\right)$. 


\section{Existence results and higher order estimates}

\subsection{Galerkin scheme}

We introduce a space-discretization of the oscillatory system (1.2). Throughout this section, the parameter $\eta>0$ is held fixed, we hence drop $\eta$ from our notation. Our estimates will not depend on $\eta$.

For an arbitrary size-parameter $h>0$ let $\mathscr{T}_{h}:=\left\{K_{q}\right\}_{q \in \Lambda_{h}}$ be a regular triangulation of $\Omega$, the sets $K_{q}$ are simplices such that $\max \left\{\operatorname{diam}\left(K_{q}\right), q \in \Lambda_{h}\right\} \leq h$ and $\Lambda_{h}$ is a finite set of indices. Let $\mathscr{P}_{k}(K)$ be the space of polynomials of degree at most $k \geq 0$ on the simplex $K$. We define the function spaces

$$
\begin{aligned}
V_{h} & :=\left\{u \in H^{1}\left(\Omega ; \mathbb{R}^{n}\right): u_{\mid K} \in \mathscr{P}_{1}\left(K ; \mathbb{R}^{n}\right) \forall K \in \mathscr{T}_{h}\right\}, \\
W_{h} & :=\left\{w \in L^{2}\left(\Omega ; \mathbb{R}^{N}\right): w_{\mid K} \in \mathscr{P}_{0}\left(K ; \mathbb{R}^{N}\right) \forall K \in \mathscr{T}_{h}\right\}, \\
Z_{h} & :=\left\{z \in L^{2}\left(\Omega ; \mathbb{R}_{s}^{n \times n}\right): z_{\mid K} \in \mathscr{P}_{0}\left(K ; \mathbb{R}_{s}^{n \times n}\right) \forall K \in \mathscr{T}_{h}\right\} .
\end{aligned}
$$

Let $P_{h}^{V}: H^{1}\left(\Omega ; \mathbb{R}^{n}\right) \rightarrow V_{h}$ be the $L^{2}$-orthogonal projection onto $V_{h}$ and let $P_{h}^{W}$ : $L^{2}\left(\Omega ; \mathbb{R}^{N}\right) \rightarrow W_{h}$ be the $L^{2}$-orthogonal projection onto $W_{h}$. For regular triangulations, we have the compatibility and approximation property of the projection (see, e.g. [5])

$$
\left\|P_{h}^{V} v\right\|_{H^{1}(\Omega)} \leq C\|v\|_{H^{1}(\Omega)}, \quad P_{h}^{V} v \rightarrow v \quad \text { strongly in } H^{1}(\Omega), \quad \forall v \in H^{1}(\Omega) .
$$

We define discretized coefficients as averages

$$
D_{h}^{-1}(x):=f_{K} D^{-1}\left(\zeta, \frac{\zeta}{\eta}\right) d \zeta, L_{h}(x):=f_{K} L\left(\zeta, \frac{\zeta}{\eta}\right) d \zeta, B_{h}(x):=f_{K} B\left(\zeta, \frac{\zeta}{\eta}\right) d \zeta
$$

for $x \in K \in \mathscr{T}_{h}$. It is immediate that the averages $D_{h}^{-1}, L_{h}, B_{h}$ satisfy the positivity and boundedness assumptions (A1), independently of $h$ and $\eta$. In particular, $D_{h}^{-1}(x)$ has an inverse (for every $x$ ), which we denote by $D_{h}(x)$. In the following we also use, by slight abuse of notation,

$$
g_{\delta}(p ; x)=g_{\delta}(p ; x, x / \eta), \quad \varrho(x)=\varrho(x, x / \eta) .
$$

Since we need test-functions that vanish on the boundary of $\Omega$, we introduce the space $V_{h, 0}:=\left\{u \in V_{h}: u=0\right.$ on $\left.\partial \Omega\right\}$.

The semi-discrete problem. The natural weak discretized formulation of problem $(1.2)$ is as follows. Find $u_{h}:[0, T] \rightarrow V_{h}, \xi_{h}:[0, T] \rightarrow W_{h}$, and $\sigma_{h}:[0, T] \rightarrow Z_{h}$ solving

$$
\begin{aligned}
\int_{\Omega} \varrho \partial_{t}^{2} u_{h}(t) \cdot \varphi & =-\int_{\Omega} \sigma_{h}(t): \nabla \varphi+\int_{\Omega} f(t) \cdot \varphi & & \forall \varphi \in V_{h, 0}, \\
\int_{\Omega} D_{h}^{-1} \sigma_{h}(t): \psi & =\int_{\Omega}\left(\nabla^{s} u_{h}(t)-B_{h} \xi_{h}(t)\right): \psi & & \forall \psi \in Z_{h}, \\
\int_{\Omega} \partial_{t} \xi_{h}(t) \cdot \zeta & =\int_{\Omega} g_{\delta}\left(B_{h}^{T} \sigma_{h}(t)-L_{h} \xi_{h}(t)\right) \cdot \zeta & & \forall \zeta \in W_{h},
\end{aligned}
$$

for all $t \in(0, T)$. For the initial conditions we use sequences $u_{0, h} \rightarrow u_{0}$ in $H^{1}(\Omega)$, $u_{1, h} \rightarrow u_{1}$ in $L^{2}(\Omega)$, and $\xi_{0, h} \rightarrow \xi_{0}$ in $L^{2}(\Omega)$. We demand

$$
\int_{\Omega}\left(u_{h}(0)-u_{0, h}\right) \cdot \varphi=0, \quad \int_{\Omega}\left(\partial_{t} u_{h}(0)-u_{1, h}\right) \cdot \varphi=0, \quad \int_{\Omega}\left(\xi_{h}(0)-\xi_{0, h}\right) \cdot \zeta=0
$$


for all $\varphi \in V_{h, 0}$ and all $\zeta \in W_{h}$. As boundary condition we demand, with $U_{h}(t):=$ $P_{h}^{V} U_{0}(t)$,

$$
u_{h}(t)-U_{h}(t) \in V_{h, 0} \quad \text { for all } t \in(0, T) .
$$

\subsection{Energy estimates}

Lemma 4.1. Let assumptions (A1)-(A3) be satisfied. Let $f \in L^{2}\left(0, T ; L^{2}\left(\Omega ; \mathbb{R}^{n}\right)\right)$ be given, as well as the initial data $u_{0} \in H^{1}\left(\Omega ; \mathbb{R}^{n}\right), u_{1} \in L^{2}\left(\Omega ; \mathbb{R}^{n}\right), \xi_{0} \in L^{2}\left(\Omega ; \mathbb{R}^{N}\right)$ and the boundary condition $U_{0} \in H^{2}\left(0, T ; L^{2}\left(\Omega ; \mathbb{R}^{n}\right)\right) \cap H^{1}\left(0, T ; H^{1}\left(\Omega ; \mathbb{R}^{n}\right)\right)$. Let $h \rightarrow 0$ be a sequence of maximal diameters of the space discretization. Then, for all $h, \delta>0$,

1. The semi-discrete problem (4.2)-(4.4) has a unique solution

$$
\left(u_{h}, \partial_{t} u_{h}, \xi_{h}, \sigma_{h}\right) \in C^{1}\left([0, T] ; V_{h} \times V_{h} \times W_{h} \times Z_{h}\right)
$$

2. For every $t \in[0, T]$, the solution satisfies the energy balance

$$
\begin{gathered}
\left\{\frac{1}{2} \int_{\Omega} \varrho\left|\partial_{t}\left(u_{h}(s)-U_{h}(s)\right)\right|^{2}+\frac{1}{2} \int_{\Omega} D_{h}^{-1} \sigma_{h}(s): \sigma_{h}(s)+\frac{1}{2} \int_{\Omega} L_{h} \xi_{h}(s) \cdot \xi_{h}(s)\right\}_{s=0}^{s=t} \\
+\int_{\Omega_{t}} \partial_{t} \xi_{h} \cdot\left(B_{h}^{T} \sigma_{h}-L_{h} \xi_{h}\right)=\int_{\Omega_{t}} f_{h} \cdot \partial_{t}\left(u_{h}-U_{h}\right)+\int_{\Omega_{t}} \sigma_{h}: \nabla^{s} \partial_{t} U_{h},
\end{gathered}
$$

with $U_{h}:=P_{h}^{V} U_{0}$ and $f_{h}:=f-\varrho \partial_{t}^{2} U_{h}$.

3. With a constant $C_{0}=C_{0}(\Omega, \alpha, \beta, T)$ which does not depend on $h, \eta$, or $\delta$, there holds the a priori estimate

$$
\begin{aligned}
\left\|u_{h}\right\|_{L^{\infty}\left(0, T ; V_{h}\right)}+\left\|\partial_{t} u_{h}\right\|_{L^{\infty}\left(0, T ; L^{2}(\Omega)\right)}+\left\|\xi_{h}\right\|_{L^{\infty}\left(0, T ; W_{h}\right)}+\left\|\sigma_{h}\right\|_{L^{\infty}\left(0, T ; Z_{h}\right)} \\
\leq C_{0}\left(\left\|u_{0}\right\|_{H^{1}\left(\Omega ; \mathbb{R}^{n}\right)}+\left\|u_{1}\right\|_{L^{2}\left(\Omega ; \mathbb{R}^{n}\right)}+\left\|\xi_{0}\right\|_{L^{2}\left(\Omega ; \mathbb{R}^{N}\right)}+\|f\|_{L^{2}\left(0, T ; L^{2}(\Omega)\right)}\right. \\
\left.+\left\|U_{0}\right\|_{H^{1}\left(0, T ; H^{1}\left(\Omega ; \mathbb{R}^{n}\right)\right)}+\left\|U_{0}\right\|_{H^{2}\left(0, T ; L^{2}\left(\Omega ; \mathbb{R}^{n}\right)\right)}\right) .
\end{aligned}
$$

Proof. Step 1: Solution of the discrete equation. By substituting the expression for $\sigma_{h}$ from (4.2b) into the other two equations, we see that the evolution system (4.2) can be written as a system of ordinary differential equations in the unknowns $u_{h} \in V_{h}$ and $\xi_{h} \in W_{h}$ :

$$
\begin{aligned}
& \int_{\Omega} \varrho \partial_{t}^{2} u_{h}(t) \cdot \varphi=-\int_{\Omega} D_{h}\left(\nabla^{s} u_{h}(t)-B_{h} \xi_{h}(t)\right): \nabla \varphi+\int_{\Omega} f(t) \cdot \varphi \quad \forall \varphi \in V_{h, 0}, \\
& \int_{\Omega} \partial_{t} \xi_{h}(t) \cdot \zeta=\int_{\Omega} g_{\delta}\left(B_{h}^{T} D_{h}\left(\nabla^{s} u_{h}(t)-B_{h} \xi_{h}(t)\right)-L_{h} \xi_{h}(t)\right) \cdot \zeta \quad \forall \zeta \in W_{h} .
\end{aligned}
$$

The right-hand side of (4.7) is Lipschitz-continuous since, owing to $(\mathrm{A} 3), g_{\delta}(\cdot ; x)$ is Lipschitz-continuous. Hence there exists a maximal time interval $\left[0, T_{\max }\right) \subset[0, T)$ such that (4.2) has a unique $C^{1}$-regular solution in $\left[0, T_{\max }\right)$. Owing to the a priori estimates that are obtained in Step 3, we conclude $T_{\max }=T$. 
Step 2: Energy balance. Since $u_{h}, U_{h} \in C^{1}\left(\left[0, T_{\max }\right) ; V_{h}\right)$ and $u_{h}(t)-U_{h}(t)=0$ on $\partial \Omega$, the function $\varphi=\partial_{t}\left(u_{h}(t)-U_{h}(t)\right) \in V_{h, 0}$ is an admissible test-function in (4.2a). Subtracting $\varrho \partial_{t}^{2} U_{h}(t)$ from both sides of (4.2a) we obtain

$$
\int_{\Omega} \varrho \partial_{t} \varphi \cdot \varphi=-\int_{\Omega} \sigma_{h}(t): \nabla^{s} \varphi+\int_{\Omega}\left(f(t)-\varrho \partial_{t}^{2} U_{h}(t)\right) \cdot \varphi .
$$

The identification as a total time derivative provides, inserting $\varphi$,

$$
\begin{aligned}
\frac{1}{2} \frac{d}{d t} \int_{\Omega} \varrho\left|\partial_{t}\left(u_{h}(t)-U_{h}(t)\right)\right|^{2}= & -\int_{\Omega} \sigma_{h}(t): \partial_{t} \nabla^{s} u_{h}(t)+\int_{\Omega} \sigma_{h}(t): \partial_{t} \nabla^{s} U_{h}(t) \\
& +\int_{\Omega}\left(f(t)-\varrho \partial_{t}^{2} U_{h}(t)\right) \cdot \partial_{t}\left(u_{h}(t)-U_{h}(t)\right) .
\end{aligned}
$$

We next use (4.2b), which we differentiate with respect to time, and use the testfunction $\psi=\sigma_{h}(t)$. We obtain for one of the above integrals

$$
\begin{aligned}
\int_{\Omega} \sigma_{h}(t): \partial_{t} \nabla^{s} u_{h}(t)=\int_{\Omega} D_{h}^{-1} \partial_{t} \sigma_{h}(t): \sigma_{h}(t)+B_{h} \partial_{t} \xi_{h}(t): \sigma_{h}(t) \\
=\frac{1}{2} \frac{d}{d t}\left\{\int_{\Omega} D_{h}^{-1} \sigma_{h}(t): \sigma_{h}(t)\right\}+\int_{\Omega} \partial_{t} \xi_{h}(t): B_{h}^{T} \sigma_{h}(t) \\
=\frac{1}{2} \frac{d}{d t}\left\{\int_{\Omega} D_{h}^{-1} \sigma_{h}(t): \sigma_{h}(t)\right\}+\int_{\Omega} \partial_{t} \xi_{h}(t):\left(B_{h}^{T} \sigma_{h}(t)-L_{h} \xi_{h}(t)+L_{h} \xi_{h}(t)\right) \\
=\frac{1}{2} \frac{d}{d t}\left\{\int_{\Omega} D_{h}^{-1} \sigma_{h}(t): \sigma_{h}(t)+\xi_{h}(t): L_{h} \xi_{h}(t)\right\} \\
\quad+\int_{\Omega} \partial_{t} \xi_{h}(t) \cdot\left(B_{h}^{T} \sigma_{h}(t)-L_{h} \xi_{h}(t)\right) .
\end{aligned}
$$

Inserting this term into (4.8) and integrating over $(0, t)$ yields the energy balance (4.5).

Step 3: Energy estimates. The monotonicity of the approximation $g_{\delta}$ of (A3) implies

$$
\int_{\Omega} \partial_{t} \xi_{h} \cdot\left(B_{h}^{T} \sigma_{h}-L_{h} \xi_{h}\right) \stackrel{(4.2 \mathrm{c})}{=} \int_{\Omega} g_{\delta}\left(B_{h}^{T} \sigma_{h}-L_{h} \xi_{h}\right) \cdot\left(B_{h}^{T} \sigma_{h}-L_{h} \xi_{h}\right) \geq 0 .
$$

The Cauchy-Schwarz inequality allows to estimate the right-hand side of the energy balance (4.5), and we obtain, since $\varrho, D_{h}^{-1}, L_{h}$ are positive and bounded,

$$
\begin{aligned}
& \alpha\left(\left\|\partial_{t} u_{h}(t)-\partial_{t} U_{h}(t)\right\|_{L^{2}\left(\Omega ; \mathbb{R}^{n}\right)}^{2}+\left\|\sigma_{h}(t)\right\|_{L^{2}\left(\Omega ; \mathbb{R}_{s}^{n \times n}\right)}^{2}+\left\|\xi_{h}(t)\right\|_{L^{2}\left(\Omega ; \mathbb{R}^{N}\right)}^{2}\right) \\
& \leq \beta\left(\left\|\partial_{t} u_{h}(0)-\partial_{t} U_{h}(0)\right\|_{L^{2}\left(\Omega ; \mathbb{R}^{n}\right)}^{2}+\left\|\sigma_{h}(0)\right\|_{L^{2}\left(\Omega ; \mathbb{R}_{s}^{n \times n}\right)}^{2}+\left\|\xi_{h}(0)\right\|_{L^{2}\left(\Omega ; \mathbb{R}^{N}\right)}^{2}\right) \\
&+\left\|f_{h}\right\|_{L^{2}\left(0, T ; L^{2}\left(\Omega ; \mathbb{R}^{n}\right)\right)}^{2}+\left\|U_{h}\right\|_{H^{1}\left(0, T ; H^{1}\left(\Omega ; \mathbb{R}^{n}\right)\right)}^{2} \\
&+\int_{0}^{t}\left\{\left\|\partial_{t} u_{h}(s)-\partial_{t} U_{h}(s)\right\|_{L^{2}\left(\Omega ; \mathbb{R}^{n}\right)}^{2}+\left\|\sigma_{h}(s)\right\|_{L^{2}\left(\Omega ; \mathbb{R}_{s}^{n \times n}\right)}^{2}\right\} d s .
\end{aligned}
$$

Regarding the initial condition for the stress, we can use the decomposition rule. Continuity of the solution $u_{h}, \sigma_{h}, \xi_{h}$ in $t=0$ allows to use equation (4.2b),

$$
\begin{aligned}
& \left\|\sigma_{h}(0)\right\|_{L^{2}\left(\Omega ; \mathbb{R}_{s}^{n \times n}\right)}^{2}=\left\|D_{h}\left(\nabla^{s} u_{h}(0)-B_{h} \xi_{h}(0)\right)\right\|_{L^{2}\left(\Omega ; \mathbb{R}_{s}^{n \times n}\right)}^{2} \\
& \quad \leq c\left(\left\|u_{0, h}\right\|_{H^{1}\left(\Omega ; \mathbb{R}^{n}\right)}^{2}+\left\|\xi_{0, h}\right\|_{L^{2}\left(\Omega ; \mathbb{R}^{N}\right)}^{2}\right) \leq c\left(\left\|u_{0}\right\|_{H^{1}\left(\Omega ; \mathbb{R}^{n}\right)}^{2}+\left\|\xi_{0}\right\|_{L^{2}\left(\Omega ; \mathbb{R}^{N}\right)}^{2}\right),
\end{aligned}
$$


for some constant $c$ that depends only on $\alpha$ and $\beta$. Similarly, the initial time derivative $\partial_{t} u_{h}(0)$ is bounded in $L^{2}(\Omega)$ by $c\left\|u_{1}\right\|_{L^{2}(\Omega)}$. By definition of $f_{h}$, there holds

$$
\left\|f_{h}\right\|_{L^{2}\left(0, T ; L^{2}\left(\Omega ; \mathbb{R}^{n}\right)\right)}^{2} \leq c\left(\|f\|_{L^{2}\left(0, T ; L^{2}\left(\Omega ; \mathbb{R}^{n}\right)\right)}^{2}+\left\|U_{h}\right\|_{H^{2}\left(0, T ; L^{2}\left(\Omega ; \mathbb{R}^{n}\right)\right)}^{2}\right) .
$$

We use Gronwall's Lemma to take care of the terms $\left|\partial_{t}\left(u_{h}-U_{h}\right)\right|^{2}$ and $\left|\sigma_{h}\right|^{2}$ in the right-hand side of (4.10) and obtain, with a constant $c=c(\alpha, \beta, T)$,

$$
\begin{array}{r}
\left\|\partial_{t} u_{h}\right\|_{L^{\infty}\left(0, T ; L^{2}\left(\Omega ; \mathbb{R}^{n}\right)\right)}+\left\|\xi_{h}\right\|_{L^{\infty}\left(0, T ; W_{h}\right)}+\left\|\sigma_{h}\right\|_{L^{\infty}\left(0, T ; Z_{h}\right)} \\
\leq c\left(\left\|u_{0}\right\|_{H^{1}\left(\Omega ; \mathbb{R}^{n}\right)}+\left\|u_{1}\right\|_{L^{2}\left(\Omega ; \mathbb{R}^{n}\right)}+\left\|\xi_{0}\right\|_{L^{2}\left(\Omega ; \mathbb{R}^{N}\right)}+\|f\|_{L^{2}\left(0, T ; L^{2}\left(\Omega ; \mathbb{R}^{n}\right)\right)}\right. \\
\left.+\left\|U_{h}\right\|_{H^{1}\left(0, T ; H^{1}\left(\Omega ; \mathbb{R}^{n}\right)\right)}+\left\|U_{h}\right\|_{H^{2}\left(0, T ; L^{2}\left(\Omega ; \mathbb{R}^{n}\right)\right)}\right)
\end{array}
$$

Since $P_{h}$ does not depend on time, we have $\left\|U_{h}\right\|_{H^{2}\left(0, T ; L^{2}\left(\Omega ; \mathbb{R}^{n}\right)\right)} \leq\left\|U_{0}\right\|_{H^{2}\left(0, T ; L^{2}\left(\Omega ; \mathbb{R}^{n}\right)\right)}$. By hypothesis (4.1) we additionally have $\left\|U_{h}\right\|_{H^{1}\left(0, T ; H^{1}\left(\Omega ; \mathbb{R}^{n}\right)\right)}$ bounded. Applying Korn's inequality to $\nabla^{s}\left(u_{h}-U_{h}\right)=D_{h}^{-1} \sigma_{h}+B_{h} \xi_{h}-\nabla^{s} U_{h}$, we estimate

$$
\begin{aligned}
\left\|u_{h}(t)\right\|_{H^{1}\left(\Omega ; \mathbb{R}^{n}\right)} & \leq\left\|u_{h}(t)-U_{h}(t)\right\|_{H^{1}\left(\Omega ; \mathbb{R}^{n}\right)}+\left\|U_{h}(t)\right\|_{H^{1}\left(\Omega ; \mathbb{R}^{n}\right)} \\
& \leq c(\Omega)\left(\left\|\nabla^{s}\left(u_{h}(t)-U_{h}(t)\right)\right\|_{L^{2}\left(\Omega ; \mathbb{R}_{s}^{n \times n}\right)}+\left\|U_{h}(t)\right\|_{H^{1}\left(\Omega ; \mathbb{R}^{n}\right)}\right) \\
& \leq c(\alpha, \beta, \Omega)\left(\left\|\sigma_{h}(t)\right\|_{L^{2}\left(\Omega ; \mathbb{R}_{s}^{n \times n}\right)}+\left\|\xi_{h}(t)\right\|_{L^{2}\left(\Omega ; \mathbb{R}^{N}\right)}+\left\|U_{0}(t)\right\|_{H^{1}\left(\Omega ; \mathbb{R}^{n}\right)}\right) .
\end{aligned}
$$

Passing to the supremum over $t \in(0, T)$ and combining with (4.11) we conclude (4.6).

\subsection{Higher order estimates}

In order to obtain better estimates, we have to be careful in the construction of initial conditions for finite $h>0$. One of the initial conditions can be chosen simply as the projection of the data, $u_{1, h}:=P_{h}^{V}\left(u_{1}\right)$. The initial conditions for $u_{0}$ and $\xi_{0}$ are more involved. We evaluate the initial stress $\sigma_{0}:=D\left(\nabla^{s} u_{0}-B \xi_{0}\right) \in L^{2}\left(\Omega, \mathbb{R}_{s}^{n \times n}\right)$ with its distributional divergence $F_{0}:=\nabla \cdot \sigma_{0} \in H^{-1}\left(\Omega, \mathbb{R}^{n}\right)$. We assumed the compatibility that $\Xi_{0}:=B^{T} \sigma_{0}-L \xi_{0}$ maps into $K_{0}$. We define $\Xi_{0, h} \in W_{h}$ with a projection as $\Xi_{0, h}:=P_{h}^{W} \Xi_{0}$. The convexity of $K_{0}$ implies that also $\Xi_{0, h}$ maps into $K_{0}$. We finally define $u_{0, h} \in V_{h}$ and $\xi_{0, h} \in W_{h}$ as the solution of the problem

$$
\begin{aligned}
-\int_{\Omega} \sigma_{0, h}: \nabla \varphi & =\int_{\Omega} F_{0} \cdot \varphi \\
\sigma_{0, h} & =D_{h}\left(\nabla^{s} u_{0, h}-B_{h} \xi_{0, h}\right), \\
\xi_{0, h} & =L_{h}^{-1}\left(B_{h}^{T} \sigma_{0, h}-\Xi_{0, h}\right),
\end{aligned}
$$

for all $\varphi \in V_{h, 0}$, with boundary condition $u_{0, h}-P_{h}^{V} U_{0}(0) \in V_{h, 0}$. For given $\xi_{0, h}$, the stationary elasticity system (4.12)-(4.13) is solvable by standard Finite Element theory, see, e.g., [5]. The corresponding map $\xi_{0, h} \mapsto-B_{h}^{T} \sigma_{0, h}$ is monotone, as a consequence, also $\xi_{0, h} \mapsto L_{h} \xi_{0, h}-B_{h}^{T} \sigma_{0, h}$ is monotone. This implies the solvability of system (4.12)-(4.14).

For a regular discretization of the domain, Finite Element theory provides $u_{0, h} \rightarrow u_{0}$ in $H^{1}(\Omega)$ for $h \rightarrow 0$, and $\sigma_{0, h} \rightarrow \sigma_{0}, \xi_{0, h} \rightarrow \xi_{0}$ in $L^{2}(\Omega)$. The construction guarantees additionally that $B_{h}^{T} \sigma_{0, h}-L_{h} \xi_{0, h}$ maps into $K_{0}$. 
Lemma 4.2 (Higher-order estimates). Let assumptions (A1)-(A3) be satisfied. For parameters $\eta, \delta, h>0$ we investigate the solutions of (4.2)-(4.4). We consider a fixed right hand side $f \in H^{1}\left(0, T ; L^{2}\left(\Omega ; \mathbb{R}^{n}\right)\right)$, initial data $u_{0}, u_{1} \in H^{1}\left(\Omega ; \mathbb{R}^{n}\right)$ and $\xi_{0} \in L^{2}\left(\Omega ; \mathbb{R}^{N}\right)$, and boundary data $U_{0} \in H^{3}\left(0, T ; L^{2}\left(\Omega ; \mathbb{R}^{n}\right)\right) \cap H^{2}\left(0, T ; H^{1}\left(\Omega ; \mathbb{R}^{n}\right)\right)$. Let $h \rightarrow 0$ be a sequence of maximal diameters of the space discretization as above.

We assume that $\sigma_{0}:=D\left(\nabla^{s} u_{0}-B \xi_{0}\right) \in L^{2}\left(\Omega, \mathbb{R}_{s}^{n \times n}\right)$ has a distributional divergence $F_{0}:=\nabla \cdot \sigma_{0} \in L^{2}\left(\Omega, \mathbb{R}^{n}\right)$. On the initial values $\xi_{0}$ we assume that $\left(B^{T} \sigma_{0}-L \xi_{0}\right)(x) \in K_{0}$ for every $x \in \Omega$. Then, with a constant $C_{0}=C_{0}(\Omega, \alpha, \beta, T)$ which does not depend on $h, \eta, \delta$, there holds the a priori estimate

$$
\begin{gathered}
\left\|\partial_{t} u_{h}\right\|_{L^{\infty}\left(0, T ; V_{h}\right)}+\left\|\partial_{t}^{2} u_{h}\right\|_{L^{\infty}\left(0, T ; L^{2}(\Omega)\right)}+\left\|\xi_{h}\right\|_{W^{1, \infty}\left(0, T ; W_{h}\right)}+\left\|\sigma_{h}\right\|_{W^{1, \infty}\left(0, T ; Z_{h}\right)} \\
\leq C_{0}\left(1+\left\|u_{0}\right\|_{H^{1}\left(\Omega ; \mathbb{R}^{n}\right)}+\left\|u_{1}\right\|_{H^{1}\left(\Omega ; \mathbb{R}^{n}\right)}+\left\|\nabla \cdot \sigma_{0}\right\|_{L^{2}(\Omega)}+\left\|\xi_{0}\right\|_{L^{2}\left(\Omega ; \mathbb{R}^{N}\right)}\right. \\
\left.+\|f\|_{H^{1}\left(0, T ; L^{2}(\Omega)\right)}+\left\|U_{0}\right\|_{H^{2}\left(0, T ; H^{1}\left(\Omega ; \mathbb{R}^{n}\right)\right)}+\left\|U_{0}\right\|_{H^{3}\left(0, T ; L^{2}\left(\Omega ; \mathbb{R}^{n}\right)\right)}\right)
\end{gathered}
$$

Proof. For simplicity, we assume in this calculation $g_{\delta}(\cdot ; x) \in C^{1}\left(\mathbb{R}^{N}\right)$, and note that the assumption can be relaxed to $g_{\delta}(\cdot ; x) \in W^{1, \infty}\left(\mathbb{R}^{N}\right)$ as in $(2.6)$, by an argument with finite differences. We adopt the same notation as in Lemma 4.1. We differentiate equation (4.2a) with respect to $t$ and use the test-function $\varphi:=\partial_{t}^{2}\left(u_{h}(t)-U_{h}(t)\right) \in V_{h, 0}$ for arbitrary $t \in(0, T)$. This provides the relation

$$
\begin{aligned}
\frac{1}{2} \frac{d}{d t} \int_{\Omega} \varrho\left|\partial_{t}^{2}\left(u_{h}(t)-U_{h}(t)\right)\right|^{2}=- & \int_{\Omega} \partial_{t} \sigma_{h}(t): \partial_{t}^{2} \nabla^{s}\left(u_{h}(t)-U_{h}(t)\right) \\
& \left.+\int_{\Omega} \partial_{t} f_{h}(t) \cdot \partial_{t}^{2}\left(u_{h}(t)-U_{h}(t)\right)\right) .
\end{aligned}
$$

We next differentiate $(4.2 \mathrm{~b})$ twice with respect to time and use $\psi:=\partial_{t} \sigma_{h}(t)$ as test-function. In the following steps, we add and subtract the term $L_{h} \partial_{t} \xi_{h}(t)$ and substitute $\partial_{t}^{2} \xi_{h}(t)$ using equation (4.2c) (differentiated in time). If we set $\Xi_{h}(t):=$ $B_{h}^{T} \sigma_{h}(t)-L_{h} \xi_{h}(t)$ and suppress the dependence on $t$, we find

$$
\begin{aligned}
\int_{\Omega} \partial_{t} \sigma_{h}: \partial_{t}^{2} \nabla^{s} u_{h}=\int_{\Omega} D_{h}^{-1} \partial_{t}^{2} \sigma_{h}: \partial_{t} \sigma_{h}+B_{h} \partial_{t}^{2} \xi_{h}: \partial_{t} \sigma_{h} \\
=\frac{1}{2} \frac{d}{d t}\left\{\int_{\Omega} D_{h}^{-1} \partial_{t} \sigma_{h}: \partial_{t} \sigma_{h}\right\}+\int_{\Omega} \partial_{t}^{2} \xi_{h}:\left(B_{h}^{T} \partial_{t} \sigma_{h}-L_{h} \partial_{t} \xi_{h}+L_{h} \partial_{t} \xi_{h}\right) \\
=\frac{1}{2} \frac{d}{d t}\left\{\int_{\Omega} D_{h}^{-1} \partial_{t} \sigma_{h}: \partial_{t} \sigma_{h}+\partial_{t} \xi_{h}: L_{h} \partial_{t} \xi_{h}\right\}+\int_{\Omega} \partial_{t}\left\{g_{\delta}\left(\Xi_{h}\right)\right\} \cdot \partial_{t} \Xi_{h} \\
=\frac{1}{2} \frac{d}{d t}\left\{\int_{\Omega} D_{h}^{-1} \partial_{t} \sigma_{h}: \partial_{t} \sigma_{h}+\partial_{t} \xi_{h}: L_{h} \partial_{t} \xi_{h}\right\}+\int_{\Omega}\left[\nabla_{\xi} g_{\delta}\left(\Xi_{h}\right) \partial_{t} \Xi_{h}\right] \cdot \partial_{t} \Xi_{h} .
\end{aligned}
$$

Owing to the monotonicity of $g_{\delta}$ (see (2.9)), the last term is nonnegative. With an integration over the interval $(0, t)$ we obtain

$$
\begin{aligned}
& \frac{1}{2}\left\{\int_{\Omega} \varrho\left|\partial_{t}^{2}\left(u_{h}(s)-U_{h}(s)\right)\right|^{2}+\int_{\Omega} D_{h}^{-1} \partial_{t} \sigma_{h}(s): \partial_{t} \sigma_{h}(s)+\int_{\Omega} L_{h} \partial_{t} \xi_{h}(s) \cdot \partial_{t} \xi_{h}(s)\right\}_{s=0}^{s=t} \\
& \leq \int_{\Omega_{t}} \partial_{t} f_{h} \cdot \partial_{t}^{2}\left(u_{h}-U_{h}\right)+\int_{\Omega_{t}} \partial_{t} \sigma_{h}: \partial_{t}^{2} \nabla^{s} U_{h} .
\end{aligned}
$$


We have to control the norms of functions in $t=0$, more precisely, of the functions $\left.\partial_{t}^{2}\left(u_{h}-U_{h}\right)\right|_{t=0},\left.\partial_{t} \sigma_{h}\right|_{t=0}$, and $\left.\partial_{t} \xi_{h}\right|_{t=0}$ in $L^{2}(\Omega)$, independently of $h$ and $\delta$. Using equation (4.2a) in $t=0$ with $\varphi=\partial_{t}^{2}\left(u_{h}(0)-U_{h}(0)\right)$ and the Cauchy-Schwarz inequality, we obtain with $f_{h}:=f-\varrho \partial_{t}^{2} U_{h}$

$$
\begin{gathered}
\int_{\Omega}\left|\partial_{t}^{2}\left(u_{h}(0)-U_{h}(0)\right)\right|^{2} \leq c\left\|f_{h}(0)\right\|_{L^{2}(\Omega)}^{2}+\int_{\Omega} \nabla\left(\partial_{t}^{2}\left(u_{h}(0)-U_{h}(0)\right)\right) \cdot \sigma_{h}(0) \\
=c\left\|f_{h}(0)\right\|_{L^{2}(\Omega)}^{2}+\int_{\Omega} \nabla\left(\partial_{t}^{2}\left(u_{h}(0)-U_{h}(0)\right)\right) \cdot D_{h}\left(\nabla^{s} u_{0, h}-B_{h} \xi_{0, h}\right) .
\end{gathered}
$$

By construction of $u_{0, h}$, the last integral is of the form $\int_{\Omega} \nabla \psi: \sigma_{0, h}=-\int_{\Omega} \psi \cdot F_{0}$, we therefore find

$$
\begin{aligned}
\int_{\Omega}\left|\partial_{t}^{2}\left(u_{h}(0)-U_{h}(0)\right)\right|^{2} & \leq c\left(\left\|f_{h}(0)\right\|_{L^{2}(\Omega)}^{2}+\left\|F_{0}\right\|_{L^{2}(\Omega)}^{2}\right) \\
& \leq c\left(\|f(0)\|_{L^{2}(\Omega)}^{2}+\left\|\partial_{t}^{2} U_{h}(0)\right\|_{L^{2}(\Omega)}^{2}+\left\|F_{0}\right\|_{L^{2}(\Omega)}^{2}\right) \\
& \leq c\left(\|f(0)\|_{L^{2}(\Omega)}^{2}+\left\|U_{0}\right\|_{H^{3}\left(0, T ; L^{2}(\Omega)\right)}^{2}+\left\|F_{0}\right\|_{L^{2}(\Omega)}^{2}\right) .
\end{aligned}
$$

The time derivative of the inner variables in $t=0$ is $\partial_{t} \xi_{h}(0)=P_{h}^{W} g_{\delta}\left(B_{h}^{T} \sigma_{0, h}-\right.$ $\left.L_{h} \xi_{0, h}\right)=: \xi_{1, h}$. We assumed that $B^{T} \sigma_{0}-L \xi_{0}$ maps into $K_{0}$, our choice of $\xi_{0, h}$ guarantees that $B_{h}^{T} \sigma_{0, h}-L_{h} \xi_{0, h}$ maps again into $K_{0}$, hence $\xi_{1, h}$ is bounded in $L^{2}(\Omega)$, independently of $h$ and $\eta$, by assumption (A3).

Differentiating (4.2b) with respect to time and testing with $\psi=\partial_{t} \sigma_{h}(0)$, we find

$$
\begin{aligned}
\int_{\Omega}\left|\partial_{t} \sigma_{h}(0)\right|^{2} & \leq \int_{\Omega}\left|D_{h}\left(\nabla^{s} \partial_{t} u_{h}(0)-B_{h} \partial_{t} \xi_{h}(0)\right)\right|^{2} \\
& \leq c\left(\int_{\Omega}\left|\nabla^{s} u_{1, h}\right|^{2}+\left|\xi_{1, h}\right|^{2}\right) \leq c\left(\left\|u_{1}\right\|_{H^{1}(\Omega)}^{2}+\left\|\xi_{1, h}\right\|_{L^{2}(\Omega)}^{2}\right) .
\end{aligned}
$$

Inserting estimates (4.18) and (4.21) into (4.17), we obtain the sought estimates on $\partial_{t}^{2} u_{h}, \partial_{t} \xi_{h}$ and $\partial_{t} \sigma_{h}$. The bound for $\partial_{t} u_{h}$ in $L^{\infty}\left(0, T ; V_{h}\right)$ follows from the latter, differentiating $(4.2 \mathrm{~b})$ with respect to time.

\subsection{Convergence of the discretized-regularized solution}

Owing to the a priori estimates of Lemma 4.2 we can find a subsequence $\left\{h_{k}, \delta_{k}\right\}_{k \in \mathbb{N}}$ and a limit vector $(u, \sigma, \xi)$ such that the following convergences hold:

$$
\begin{aligned}
\partial_{t}^{2} u_{k} & \rightarrow \partial_{t}^{2} u, & & \text { weakly in } L^{2}\left(\Omega_{T} ; \mathbb{R}^{n}\right), \\
\partial_{t} u_{k} & \rightarrow \partial_{t} u, & & \text { weakly in } H^{1}\left(\Omega_{T} ; \mathbb{R}^{n}\right), \\
\partial_{t} \xi_{k} & \rightarrow \partial_{t} \xi, & & \text { weakly in } L^{2}\left(\Omega_{T} ; \mathbb{R}^{N}\right), \\
\partial_{t} \sigma_{k} & \rightarrow \partial_{t} \sigma, & & \text { weakly in } L^{2}\left(\Omega_{T} ; \mathbb{R}_{s}^{n \times n}\right), \\
\nabla^{s} u_{k} & \rightarrow \nabla^{s} u, & & \text { weakly in } L^{2}\left(\Omega_{T} ; \mathbb{R}_{s}^{n \times n}\right) .
\end{aligned}
$$

It remains to show that the limit $(u, \sigma, \xi)$ is a solution to problem (1.2). By weak convergence, we immediately obtain that $(1.2 \mathrm{a})$ and $(1.2 \mathrm{~b})$ are satisfied. If $g$ were the subdifferential of a convex function, we could cite [25, Section 2.3] to conclude. For the more general class of operators considered here, we firstly claim the following (a 
statement with similarities to assumption (A4)): For every weakly convergent sequence $f_{\delta} \rightarrow f$ in $L^{2}\left(\Omega_{T} ; \mathbb{R}^{N}\right)$ with $h_{\delta}:=g_{\delta}\left(f_{\delta}\right) \rightarrow h$ in $L^{2}\left(\Omega_{T} ; \mathbb{R}^{N}\right)$, there holds:

$$
\liminf _{\delta \rightarrow 0} \int_{\Omega_{T}} h_{\delta} \cdot f_{\delta} \leq \int_{\Omega_{T}} h \cdot f \quad \text { implies } \quad h \in g(f) .
$$

Property (4.27) follows from assumptions (A2) and (A3), the argument is similar to that of Lemma 3.4: Let $f_{0}, h_{0} \in L^{2}\left(\Omega_{T}\right)$ be arbitrary with $h_{0} \in g\left(f_{0}\right)$, and let $f_{0, \delta}$ and $h_{0, \delta}=g_{\delta}\left(f_{0, \delta}\right)$ be $L^{2}\left(\Omega_{T}\right)$-approximations as in (2.8). Then

$$
0 \leq \int_{\Omega_{T}}\left(h_{0, \delta}-h_{\delta}\right) \cdot\left(f_{0, \delta}-f_{\delta}\right)
$$

holds by monotonicity of $g_{\delta}$. Taking the limes inferior in this expression is possible by the prerequisite of (4.27). We obtain

$$
0 \leq \int_{\Omega_{T}}\left(h_{0}-h\right) \cdot\left(f_{0}-f\right) .
$$

Since $f_{0}$ and $h_{0}$ are arbitrary, the maximal monotonicity of $g_{\Omega_{T}}$ implies $h \in g(f)$ and hence the implication (4.27).

We want to conclude that the limit functions, as $k \rightarrow \infty$, satisfy the flow rule. To this end we consider (4.5) and take the limes superior,

$$
\begin{aligned}
\limsup & \int_{\Omega_{T}} \partial_{t} \xi_{k} \cdot\left(B_{k}^{T} \sigma_{k}-L_{k} \xi_{k}\right) \\
& \stackrel{(4.5)}{\leq} \int_{\Omega_{T}} \partial_{t} u \cdot f-\int_{\Omega_{T}} \varrho \partial_{t}^{2} u \cdot \partial_{t} u-\int_{\Omega_{T}} \partial_{t} \sigma: D^{-1} \sigma-\int_{\Omega_{T}} \partial_{t} \xi \cdot L \xi \\
& \stackrel{(1.2 \mathrm{a})}{=}-\int_{\Omega_{T}} \partial_{t} u \cdot(\nabla \cdot \sigma)-\int_{\Omega_{T}} \partial_{t} \sigma: D^{-1} \sigma-\int_{\Omega_{T}} \partial_{t} \xi \cdot L \xi \\
& =\int_{\Omega_{T}} \partial_{t} \nabla^{s} u: \sigma-\int_{\Omega_{T}} \partial_{t} \sigma: D^{-1} \sigma-\int_{\Omega_{T}} \partial_{t} \xi \cdot L \xi \\
& =\int_{\Omega_{T}} \partial_{t}\left(\nabla^{s} u-D^{-1} \sigma\right): \sigma-\int_{\Omega_{T}} \partial_{t} \xi \cdot L \xi \\
& \stackrel{(1.2 \mathrm{~b})}{=} \int_{\Omega_{T}} \partial_{t}(B \xi): \sigma-\int_{\Omega_{T}} \partial_{t} \xi \cdot L \xi=\int_{\Omega_{T}} \partial_{t} \xi \cdot\left(B^{T} \sigma-L \xi\right) .
\end{aligned}
$$

We read this inequality as a property of the two functions $f_{k}:=B_{k}^{T} \sigma_{k}-L_{k} \xi_{k}$ and $h_{k}:=\partial_{t} \xi_{k}$ which satisfy $h_{k} \in g_{\delta_{k}}\left(f_{k}\right)$. Relation (4.27) provides the flow-rule $h \in g(f)$, i.e. $(1.2 \mathrm{c})$.

With this observation, the existence result and the a priori estimates of Theorem 2.1 are obtained.

\section{References}

[1] H.-D. Alber. Materials with memory, volume 1682 of Lecture Notes in Mathematics. Springer-Verlag, Berlin, 1998. Initial-boundary value problems for constitutive equations with internal variables. 
[2] H.-D. Alber. Evolving microstructure and homogenization. Contin. Mech. Thermodyn., 12(4):235-286, 2000.

[3] H.-D. Alber and S. Nesenenko. Justification of homogenization in viscoplasticity: From convergence on two scales to an asymptotic solution in $L^{2}(\Omega)$. J. Multiscale Modelling, 1:223-244, 2009.

[4] G. Allaire. Homogenization and two-scale convergence. SIAM J. Math. Anal., 23(6):1482-1518, 1992.

[5] D. Braess. Finite elements. Cambridge University Press, Cambridge, second edition, 2001. Theory, fast solvers, and applications in solid mechanics, Translated from the 1992 German edition by Larry L. Schumaker.

[6] A. Braides, V. Chiadò Piat, and A. Defranceschi. Homogenization of almost periodic monotone operators. Ann. Inst. H. Poincaré Anal. Non Linéaire, 9(4):399432, 1992.

[7] A. Braides and A. Defranceschi. Homogenization of multiple integrals, volume 12 of Oxford Lecture Series in Mathematics and its Applications. The Clarendon Press Oxford University Press, New York, 1998.

[8] H. Brezis. Opérateurs maximaux monotones et semi-groupes de contractions dans les espaces de Hilbert. North Holland, 1973.

[9] C. Castaing and M. Valadier. Convex analysis and measurable multifunctions. Lecture Notes in Mathematics, Vol. 580. Springer-Verlag, Berlin, 1977.

[10] V. Chiadò Piat and A. Defranceschi. Homogenization of monotone operators. Nonlinear Anal., 14(9):717-732, 1990.

[11] D. Cioranescu, A. Damlamian, and G. Griso. The periodic unfolding method in homogenization. SIAM J. Math. Anal., 40(4):1585-1620, 2008.

[12] A. Damlamian, N. Meunier, and J. Van Schaftingen. Periodic homogenization for convex functionals using Mosco convergence. Ric. Mat., 57(2):209-249, 2008.

[13] G. A. Francfort and A. Giacomini. On periodic homogenization in perfect elastoplasticity. J. Eur. Math. Soc., to appear.

[14] G. A. Francfort, F. Murat, and L. Tartar. Homogenization of monotone operators in divergence form with $x$-dependent multivalued graphs. Ann. Mat. Pura Appl. (4), 188(4):631-652, 2009.

[15] W. Han and B. D. Reddy. Plasticity, volume 9 of Interdisciplinary Applied Mathematics. Springer-Verlag, New York, 1999. Mathematical theory and numerical analysis.

[16] A. Mielke. Evolution of rate-independent systems. In Evolutionary equations. Vol. II, Handb. Differ. Equ., pages 461-559. Elsevier/North-Holland, Amsterdam, 2005. 
[17] A. Mielke, T. Roubíček, and U. Stefanelli. $\Gamma$-limits and relaxations for rateindependent evolutionary problems. Calc. Var. Partial Differential Equations, 31(3):387-416, 2008.

[18] A. Mielke and F. Theil. On rate-independent hysteresis models. NoDEA Nonlinear Differential Equations Appl., 11(2):151-189, 2004.

[19] A. Mielke and A. M. Timofte. Two-scale homogenization for evolutionary variational inequalities via the energetic formulation. SIAM J. Math. Anal., 39(2):642668 (electronic), 2007.

[20] S. Nesenenko. Homogenization in viscoplasticity. SIAM J. Math. Anal., 39(1):236-262, 2007.

[21] S. Nesenenko and P. Neff. Homogenization for dislocation based gradient viscoplasticity. Technical Report arXiv:1301.2911, Arxiv, 2013.

[22] G. Nguetseng. A general convergence result for a functional related to the theory of homogenization. SIAM J. Math. Anal., 20(3):608-623, 1989.

[23] B. Schweizer. Averaging of flows with capillary hysteresis in stochastic porous media. European J. Appl. Math., 18(3):389-415, 2007.

[24] B. Schweizer. Homogenization of the Prager model in one-dimensional plasticity. Contin. Mech. Thermodyn., 20(8):459-477, 2009.

[25] B. Schweizer and M. Veneroni. Periodic homogenization of the Prandtl-Reuss model with hardening. J. Multiscale Modelling, 2:69-106, 2010.

[26] M. Veneroni. Stochastic homogenization of subdifferential inclusions via scale integration. Intl. J. of Struct. Changes in Solids, 3(1):83-98, 2011.

[27] A. Visintin. Some properties of two-scale convergence. Atti Accad. Naz. Lincei Cl. Sci. Fis. Mat. Natur. Rend. Lincei (9) Mat. Appl., 15(2):93-107, 2004.

[28] A. Visintin. On homogenization of elasto-plasticity. J. Phys.: Conf. Ser., 22:222$234,2005$.

[29] A. Visintin. Homogenization of the nonlinear Kelvin-Voigt model of viscoelasticity and of the Prager model of plasticity. Contin. Mech. Thermodyn., 18(3-4):223$252,2006$.

[30] A. Visintin. Two-scale convergence of some integral functionals. Calc. Var. Partial Differential Equations, 29(2):239-265, 2007.

[31] A. Visintin. Homogenization of nonlinear visco-elastic composites. J. Math. Pures Appl. (9), 89(5):477-504, 2008.

[32] A. Visintin. Homogenization of the nonlinear Maxwell model of viscoelasticity and of the Prandtl-Reuss model of elastoplasticity. Proc. Roy. Soc. Edinburgh Sect. A, 138(6):1363-1401, 2008. 
Homogenization of plasticity equations with two-scale convergence methods

$2013-11$

$2013-10$

2013-09

$2013-08$

2013-07

2013-06

$2013-05$

2013-04

2013-03

2013-02

$2013-01$

$2012-19$

\section{$2012-18$}

$2012-17$

$2012-16$

$2012-15$

Sven Glaser

A law of large numbers for the power variation of fractional Lévy processes

Christian Palmes and Jeannette H.C. Woerner

The Gumbel test for jumps in stochastic volatility models

Agnes Lamacz, Stefan Neukamm, and Felix Otto

Moment bounds for the corrector in stochastic homogenization of a percolation model

Frank Klinker

Connections on Cahen-Wallach spaces

Andreas Rätz and Matthias Röger

Symmetry breaking in a bulk-surface reaction-diffusion model for signaling networks

\section{Gilles Francfort and Ben Schweizer}

A doubly non-linear system in small-strain visco-plasticity

\section{Tomáš Dohnal}

Traveling Solitary Waves in the Periodic Nonlinear Schrödinger Equation with Finite Band Potentials

Karl Friedrich Siburg, Pavel Stoimenov, and Gregor N.F. Weiß

Forecasting Portfolio-Value-at-Risk with Nonparametric Lower Tail Dependence Estimates

Martin Heida

On thermodynamics of fluid interfaces

Martin Heida

Existence of soulutions for two types of generalized versions

of the Cahn-Hilliard equation

T. Dohnal, A. Lamacz, B. Schweizer

Dispersive effective equations for waves in heterogeneous media on large time scales

Martin Heida

On gradient flows of nonconvex functionals in Hilbert spaces with

Riemannian metric and application to Cahn-Hilliard equations

R.V. Kohn, J. Lu, B. Schweizer, and M.I. Weinstein

A variational perspective on cloaking by anomalous localized resonance

Margit Rösler and Michael Voit

Olshanski spherical functions for infinite dimensional motion groups of fixed rank

Selim Esedō̄lu, Andreas Rätz, Matthias Röger

Colliding Interfaces in Old and New Diffuse-interface Approximations of Willmore-flow

Patrick Henning, Mario Ohlberger and Ben Schweizer

An adaptive multiscale finite element method 


\section{Alexander Schnurr}

Generalization of the Blumenthal-Getoor Index to the Class of Homogeneous

Diffusions with Jumps and some Applications

$2012-11$

$2012-10$

2012-09

2012-08

2012-07

2012-06

2012-05

2012-04

2012-03

2012-02

2012-01

2011-14

2011-13

2011-12

2011-11

\section{Wilfried Hazod}

Remarks on pseudo stable laws on contractible groups

\section{Waldemar Grundmann}

Limit theorems for radial random walks on Euclidean spaces of high dimensions

\section{Martin Heida}

A two-scale model of two-phase flow in porous media ranging from porespace to the macro scale

\section{Martin Heida}

On the derivation of thermodynamically consistent boundary conditions for the Cahn-Hilliard-Navier-Stokes system

\section{Michael Voit}

Uniform oscillatory behavior of spherical functions of $G L_{n} / U_{n}$ at the identity and a central limit theorem

\section{Agnes Lamacz and Ben Schweizer}

Effective Maxwell equations in a geometry with flat rings of arbitrary shape

\section{Frank Klinker and Günter Skoruppa}

Ein optimiertes Glättungsverfahren motiviert durch eine technische Fragestellung

Patrick Henning, Mario Ohlberger, and Ben Schweizer

Homogenization of the degenerate two-phase flow equations

Andreas Rätz

A new diffuse-interface model for step flow in epitaxial growth

Andreas Rätz and Ben Schweizer

Hysteresis models and gravity fingering in porous media

Wilfried Hazod

Intrinsic topologies on H-contraction groups with applications to semistability

Guy Bouchitté and Ben Schweizer

Plasmonic waves allow perfect transmission through sub-wavelength metallic gratings

Waldemar Grundmann

Moment functions and Central Limit Theorem for Jacobi hypergroups on $[0, \infty[$

J. Koch, A. Rätz, and B. Schweizer

Two-phase flow equations with a dynamic capillary pressure

\section{Michael Voit}

Central limit theorems for hyperbolic spaces and Jacobi processes on $[0, \infty[$ 